\title{
Explaining Xenon-1T signal with FIMP dark matter and neutrino mass in a $U(1)_{X}$ extension
}

\author{
Sarif Khan ${ }^{\mathrm{a}}(\mathbb{0}$ \\ Institut für Theoretische Physik, Georg-August-Universität Göttingen, Friedrich-Hund-Platz 1, 37077 Göttingen, Germany
}

Received: 21 April 2021 / Accepted: 29 June 2021 / Published online: 9 July 2021

(C) The Author(s) 2021

\begin{abstract}
In the present work, we have extended the standard model by an abelian $U(1)_{X}$ gauge group and additional particles. In particular, we have extended the particle content by three right handed neutrinos, two singlet scalars and two vectors like leptons. Charged assignments under different gauge groups are such that the model is gauge anomaly free and the anomaly contributions cancel among generations. Once the symmetry gets broken then three physical Higgses are produced, one axion like particle (ALP), which also acts as the $\mathrm{keV}$ scale FIMP dark matter is produced and the remaining component is absorbed by the extra gauge boson. Firstly, we have successfully generated neutrino mass by the type-I seesaw mechanism for normal hierarchy with the $3 \sigma$ bound on the oscillation parameters. The ALP in the present model can explain the Xenon-1T electron recoil signal at $\mathrm{keV}$ scale through its coupling with the electron. We have shown that ALP can be produced from the right handed neutrino decay through the freeze in mechanism. Electron and tauon get masses from dimensional-5 operators at the Planck scale and if we consider the vevs $v_{1,2} \simeq 10^{12} \mathrm{GeV}$ then we can obtain the correct value of the electron mass but not the tauon mass. The vector like leptons help in getting the correct value of the tauon mass through another higher dimensional operator which also has a role in DM production by the $2 \rightarrow 2$ process, giving the correct ballpark value of relic density for suitable reheat temperature of the Universe. We have shown that the ALP production by the higher dimensional operator can explain the electron, tauon mass and Xenon-1T signal simultaneously whereas the decay production can not explain all of them together.
\end{abstract}

\section{Introduction}

The standard model (SM) is a very successful theory in describing nature without any doubt. Although its tremen-

\footnotetext{
a e-mail: sarif.khan@uni-goettingen.de (corresponding author)
}

dous success, the SM has few flaws which can not be addressed within its particle content and gauge structure. The most noticeable limitations of the SM are the absence of a suitable dark matter (DM) candidate and neutrino masses. The presence of DM is a very well established phenomenon and has been confirmed by many experiments namely Galaxy rotation curve [1], Bullet cluster [2,3], gravitational lensing [4] and the measurements of the Cosmic Microwave Background (CMB) $[5,6]$. The satellite borne CMB experiments, WMAP [5] and Planck [6] have measured the DM relic density $\left(\Omega h^{2}\right)$ with an unprecedented accuracy which is,

$0.1172 \leq \Omega h^{2} \leq 0.1226$.

Moreover, in the SM neutrinos are massless, but from oscillation experiments, it is well established that neutrinos are massive in order to explain the flavour oscillation among the different flavours [7-17]. After a rigorous search of beyond SM signal at the collider, direct detection and indirect detection experiments, finally, Xenon-1T collaboration has announced the detection of beyond SM signal in the searches of new physics with low-energy electronic recoil data. They observed excess events over the known backgrounds in 1$7 \mathrm{keV}$ range [18]. The Xenon-1T experiment consisting of $1042 \mathrm{~kg}$ of cylindrical fiducial volume with 226.9 active days i.e. in total 0.65 tonne-year exposure, has observed 285 electron recoil events in the range $1-7 \mathrm{keV}$ in compared to the expected $232 \pm 15$ background events, which gives $3.3 \sigma$ Poissonian fluctuation. Xenon-1T considered many background models as listed in [18], among them, the prominent ones are the $\mathrm{Pb}, \mathrm{Kr}, \mathrm{Xe}, \mathrm{I}$ and solar neutrinos in the region of interest (ROI) $1-210 \mathrm{keV}$. With the 0.65 tonne-year exposure of SR 1 they can not explain the excess events. There are a couple of possible scenarios discussed in [18] which can explain the above excess namely solar axion, neutrino with the magnetic moment and bosonic dark matter. The background model is rejected at the $3.5 \sigma, 3.2 \sigma$ and $3 \sigma$ for the solar axion, neutrino magnetic moment and bosonic dark matter model, 
respectively. The parameter space needed to explain Xenon1T signal for solar axion and neutrino magnetic moment model is in strong tension with the stellar cooling [19-23] and white dwarf [24-26], globular cluster [27], respectively. Moreover, if one considers the tritium background which is neither confirmed nor excluded, then the significance of solar axion model is reduced to $2.1 \sigma$ and the neutrino magnetic moment model to $0.9 \sigma$.

In the present work, we are going to explain the signal by an axion like particle (ALP), $a$, which will be pseudo Nambu Goldstone boson (PNGB) produced due to a Peccei-Quinn type global symmetry breaking. In the present case, ALP is a suitable dark matter candidate. In the previous studies, it has been shown that ALP can be production by the thermal and misalignment mechanism. In this work, we have produced the ALP by the freeze-in mechanism and assume that ALP is produced sub-dominantly by the thermal and misalignment mechanism. The general structure of the Lagrangian of this kind of ALP is,

$$
\begin{aligned}
\mathcal{L}_{A L P}= & \left(\partial_{\mu} a\right)^{2}-m_{a}^{2} a^{2} \\
& +\frac{g_{a e e}}{2 m_{e}}\left(\partial_{\mu} a\right) \bar{e} \gamma^{\mu} \gamma_{5} e+\frac{g_{a \gamma \gamma}}{4} a F_{\mu \nu} \tilde{F}^{\mu \nu} .
\end{aligned}
$$

By using the relation $\partial_{\mu}\left(\bar{e} \gamma^{\mu} \gamma_{5} e\right)=2 i m_{e} \bar{e} \gamma_{5} e$, we can write the above equation in the following way,

$$
\begin{aligned}
\mathcal{L}_{A L P}= & \left(\partial_{\mu} a\right)^{2}-m_{a}^{2} a^{2} \\
& +\frac{g_{a \gamma \gamma}}{4} a F_{\mu \nu} \tilde{F}^{\mu \nu}+i g_{a e e} a \bar{e} \gamma_{5} e .
\end{aligned}
$$

As shown in [28], the best fit values for explaining the Xenon1T signal by the ALP are the following,

$m_{a}=2.5 \mathrm{keV}, \quad g_{a e e}=2.5 \times 10^{-14}$.

To put the constraints on the dimensionless parameters, we can redefine the couplings in the following way,

$g_{a \gamma \gamma}=\frac{\alpha_{e m}}{2 \pi V} g_{\text {eff }}^{a \gamma \gamma}, \quad g_{a e e}=\frac{m_{e}}{V} g_{e f f}^{a e e}$

where $V$ is the ALP decay constant, $g_{\text {eff }}^{a \gamma \gamma}$ and $g_{\text {eff }}^{a e e}$ are the effective coupling of axion to $\gamma \gamma$ and $e e$, respectively. We can put constraint on the $g_{\text {eff }}^{a \gamma \gamma}$ parameter from the cosmic Xray background $(\mathrm{CXB})$ for $\mathrm{keV}$ scale $\mathrm{DM}$ candidate (which corresponds to the frequency $2.98 \times 10^{17} \mathrm{~Hz}$ ) as follows [29],

$\frac{g_{\text {eff }}^{a \gamma \gamma}}{g_{\text {eff }}^{\text {aee }}} \leq 3.1 \times 10^{-3}\left(\frac{2.5 \mathrm{keV}}{m_{a}}\right)^{3 / 2} \times\left(\frac{2.7 \times 10^{-14}}{g_{\text {aee }}}\right)$

In the literature, there are extensive studies to explain the Xenon-1T signal by considering DM and neutrino magnetic moments as its origin can be found in the Refs. [30-80]. The explanations of the Xenon-1T signal mentioned earlier have considered ALP dark matter, boosted dark matter and inelastic dark matter. In Ref. [81], authors have shown that Xenon1T signal can also be explained by chameleon-screened dark energy. In the case of Xenon-1T explanation by the ALP dark matter, it is produced by thermal and misalignment mechanism. In the present case, ALP DM has been produced by the freeze-in mechanism. Moreover, Xenon-1T signal explanation by the ALP dark matter (produced by thermal and misalignment mechanism) and vector like leptons has been shown in [56] but our study is different and considering the ALP production by the freeze-in mechanism. To accomplish the dark matter, neutrino mass and the general ALP model described above (as shown in Eq. (3)), we have extended SM by additional particles and gauge group. In particular, we have extended the SM gauge structure by an additional local gauge group $U(1)_{X}$, three right handed neutrinos, two singlet scalars and two vector like leptons doublet. We have assigned the gauge charges to all the particles (SM as well as beyond SM particles) in such a way so that the gauge anomaly cancels automatically. For the Higgs doublet among four degrees of freedom (d.o.f), three of them are absorbed by the $W^{ \pm}, Z$ bosons and the remaining one is the SM Higgs. The additional four d.o.f for the two singlet scalars, one of them absorbed by the extra gauge boson present due to the additional gauge group, one of them acts as the axion like particle (ALP) and the remaining two become physical Higgses. Since the main motive apart from explaining the neutrino mass is to explain the Xenon-1T signal from DM point of view, in the present work, ALP (denoted as $a$ ) which is also feebly interacting massive particle (FIMP) dark matter takes a significant role in the phenomenology. Physical Higgses (defined as $h, h_{1}$ and $h_{2}$ ) do not actively take part in the phenomenology we are interested in this work. The charges of the two singlet Higgses are assigned in such a way that the ALP is massless and it can achieve mass from the higher dimensional operator at the Planck scale. We consider a global symmetry, $U(1)_{g}$, like Peccei-Quinn symmetry and ALP is produced when the global symmetry gets broken at the intermediate scale and the extra singlet scalars take vevs. The ALP coupling to two electrons also appears when the global symmetry breaks and it is necessary to explain the Xenon-1T signal of electron recoil at $\mathrm{keV}$ range. We assign the global charges to the particles in such a way so that $U(1)_{g} \times U(1)_{e m} \times U(1)_{e m}$ is anomaly free. Because of this anomaly cancellation, the ALP coupling to two photons is suppressed and can evade the present day strong bound from cosmic X-ray bound (CXB) [29]. Although in the present work, we are not discussing collider phenomenology of the vector like lepton [84], still it takes an important role in the production of the ALP by the freeze-in mechanism. We can produce the ALP by making the associated Yukawa coupling strength of ALP with the vector like lepton very small which is $\mathcal{O}\left(10^{-8}\right)$. Moreover, the vector like lepton is heavy compared to the associated particles and we can have dimension-5 operator after eliminating the vector like lepton, which can also produce DM by the $2 \rightarrow 2$ process for suitable values of the reheat temperature $[82,83]$. 
One major drawback for producing DM by the freeze-in mechanism is that the produced ALP DM is hard to detect at different experiments due to its feeble coupling with others. In this work, we have shown that ALP DM produced by the freeze-in mechanism can also explain the Xenon-1T signal observed recently. The coupling which helps in producing ALP DM is proportional to $\lambda_{i}$ (where $i=1,3$ and defined in Eq. (48)) and the ALP coupling to electrons is also proportional to $\lambda_{i}$, so they are interrelated. In this work, we have shown that we need heavy vector like lepton to explain everything together. The phenomenology of heavy vector like lepton to explain the lepton flavour violation processes $\left(l_{i} \rightarrow l_{j} \gamma\right.$ and $l_{i} \rightarrow 3 l$ ) has been studied in Ref. [84]. In Refs. [84-87], authors have studied $(g-2)_{\mu}$, electroweak precision data and lepton flavour violation processes by extending SM with the vector like leptons. Based on their study, vector like leptons having mass greater than a few $\mathrm{TeV}$ are safe from the aforementioned bounds. Moreover, in the present work vector like leptons are not coupled to muon generation which makes this model even less constrained than them. Therefore, we plan to study the exact bound on the model parameters from $(g-2)_{e, \mu}$, electroweak precision data and lepton flavour violation in future. Moreover, we need a high value of the singlet scalars vev to get keV ALP dark matter which makes the $\mathrm{TeV}$ scale extra gauge boson very feeble and consequently difficult to observe at the collider.

Rest of the paper is organised in the following way. In Sect. 2, we have discussed the present model in detail. Neutrino mass and allowed parameter space after satisfying oscillation have been covered in Sect. 3. Section 4 focuses on the Xenon-1T signal and production of dark matter by the freeze in mechanism. Finally we conclude in Sect. 5.

\section{Model}

In the present work, we have considered a $U(1)_{X}$ extension of the SM gauge group. Besides, the gauge group, SM particle has also been extended by three right handed neutrino, one pair of vector like leptons and two singlet scalars. All the particles are charged under the extra gauge group and in particular, they are charged in such a way so that gauge anomaly is absent. In Tables 1 and 2, we have shown the SM particles and beyond SM particles with the corresponding charges under the complete gauge group $S U(3)_{c} \times S U(2)_{L} \times U(1)_{Y} \times U(1)_{X}$.

The complete Lagrangian for the above particle spectrum which consist of kinetic term, Yukawa term and potential is as follows,

$$
\begin{aligned}
\mathcal{L}= & \mathcal{L}_{\text {kin }}+\mathcal{L}_{\text {lepton }}+\mathcal{L}_{\text {kin }}^{\phi_{i}}+\mathcal{L}_{N}+\left|D_{\mu} \phi_{h}\right|^{2} \\
& +y_{i j}^{u} Q_{i} U_{j}^{c} \bar{\phi}_{h}+y_{i j}^{d} Q_{i} D_{j}^{c} \phi_{h}+y_{1}^{V L} V L_{l 1} E_{1}^{c} \phi_{h}
\end{aligned}
$$

$$
\begin{aligned}
& +y_{3}^{V L} V L_{l 3} E_{3}^{c} \phi_{h}+y_{1}^{\phi_{1}} \phi_{1} L_{e} V L_{l 1}^{c}+y_{3}^{\phi_{1}} \bar{\phi}_{1} L_{\tau} V L_{l 3}^{c} \\
& -M_{V L_{l 1}} V L_{l 1} V L_{l 1}^{c}-M_{V L_{l 3}} V L_{l 3} V L_{l 3}^{c} \\
& -\mathcal{V}\left(\phi_{h}, \phi_{1}, \phi_{2}\right)
\end{aligned}
$$

where $\mathcal{L}_{\text {kin }}$ is the kinetic term for all the fermions and has the general form $\mathcal{L}_{k i n}=\bar{f} \gamma_{\mu} D_{\mu} f$, f is the corresponding fermion and $D_{\mu}$ is the covariant derivative with different form depending on the gauge charges of fermion $f$. $\mathcal{L}_{\text {lep }}$ contains the Yukawa terms associated with the leptons and further discussion on it is given in Eq. (37) in Sect. 4. $\mathcal{L}_{k i n}^{\phi_{i}}$ is the kinetic term for the extra singlet scalars $\phi_{i}(\mathrm{i}=1,2)$ and $U(1)_{X}$ gauge boson $Z^{\prime}$ as shown in Eq. (27). $\mathcal{L}_{N}$ is the Lagrangian associated with the Dirac neutrino mass of the neutrinos and the Majorana mass term for the right handed neutrinos,

$$
\begin{aligned}
\mathcal{L}_{N}= & y_{e e} L_{e} \phi_{h} N_{e}^{c}+y_{\mu \mu} L_{\mu} \phi_{h} N_{\mu}^{c}+y_{\tau \tau} L_{\tau} \phi_{h} N_{\tau}^{c} \\
& +y_{e \mu}^{\phi_{2}} L_{e} \phi_{h} N_{\mu}^{c} \frac{\phi_{2}}{M_{P l}}+y_{\mu e}^{\phi_{2}} L_{\mu} \phi_{h} N_{e}^{c} \frac{\phi_{2}^{\dagger}}{M_{P l}} \\
& +y_{\mu \tau}^{\phi_{2}} L_{\mu} \phi_{h} N_{\tau} \frac{\phi_{2}}{M_{P l}}+y_{\tau \mu}^{\phi_{2}} L_{\tau} \phi_{h} N_{\mu}^{c} \frac{\phi_{2}^{\dagger}}{M_{P l}} \\
& +Y_{e \mu} N_{e}^{c} N_{\mu}^{c} \phi_{2}^{\dagger}+M_{e \tau} N_{e}^{c} N_{\tau}^{c}+M_{\mu \mu} N_{\mu}^{c} N_{\mu}^{c} \\
& +Y_{\mu \tau} N_{\mu}^{c} N_{\tau}^{c} \phi_{2}+\text { h.c. }
\end{aligned}
$$

The potential for the present model takes the following form,

$$
\begin{aligned}
& \mathcal{V}\left(\phi_{h}, \phi_{1}, \phi_{2}\right)=-\mu_{h}^{2}\left(\phi_{h}^{\dagger} \phi_{h}\right)+\lambda_{h}\left(\phi_{h}^{\dagger} \phi_{h}\right)^{2}-\mu_{i}^{2}\left(\phi_{i}^{\dagger} \phi_{i}\right) \\
& \quad+\sum_{i=1,2} \lambda_{\phi_{i}}\left(\phi_{i}^{\dagger} \phi_{i}\right)^{2}+\lambda_{12}\left(\phi_{1}^{\dagger} \phi_{1}\right)\left(\phi_{2}^{\dagger} \phi_{2}\right) \\
& \quad+\sum_{i=1,2} \lambda_{\phi_{h} \phi_{i}}\left(\phi_{h}^{\dagger} \phi_{h}\right)\left(\phi_{i}^{\dagger} \phi_{i}\right)+\left[\frac{\lambda_{\phi_{1} \phi_{2}} \phi_{1}^{n} \phi_{2}^{\dagger}}{M_{P l}^{n-3}}+\text { h.c. }\right]
\end{aligned}
$$

Scalars take the following form at the time of the symmetry breaking

$$
\begin{gathered}
\phi_{h}=\left(\begin{array}{c}
0 \\
\frac{H+v}{\sqrt{2}}
\end{array}\right), \quad \phi_{1}=\left(\frac{H_{1}+v_{1}}{\sqrt{2}}\right) e^{i \frac{a_{1}}{v_{1}}} \\
\text { and } \phi_{2}=\left(\frac{H_{2}+v_{2}}{\sqrt{2}}\right) e^{i \frac{a_{2}}{v_{2}}} .
\end{gathered}
$$

In the above equation we have shown the Higgses after taking vevs; in particular we have written the SM Higgs doublet in the Unitary gauge and for the other two singlets with their $\mathrm{CP}$ odd components. Due to the higher dimensional operator of the singlet Higgses at the Planck scale, the CP odd components will mix among each other and one of them is absorbed by the extra gauge boson and the remaining one will act as keV scale ALP, which is also FIMP dark matter in the present model as will be discussed in the later part of the manuscript. From the scalar potential, we can determine the tadpole free conditions and the Higgs's masses, which are as 
Table 1 SM particles and their corresponding charges under complete gauge group

\begin{tabular}{lllllllllll}
\hline Gauge group & Fermions & \multicolumn{1}{l}{ Scalars } \\
\cline { 2 - 7 } & $Q_{i}$ & $U_{i}^{c}$ & $D_{i}^{c}$ & $L_{e}$ & $L_{\mu}$ & $L_{\tau}$ & $E_{e}^{c}$ & $E_{\mu}^{c}$ & $E_{\tau}^{c}$ \\
\hline $\mathrm{SU}(3)_{c}$ & 3 & $\overline{3}$ & $\overline{3}$ & 1 & 1 & 1 & 1 & 1 & 1 \\
$\mathrm{SU}(2)_{\mathrm{L}}$ & 2 & 1 & 1 & 2 & 2 & 2 & 1 & 1 & 1 & 1 \\
$U(1)_{Y}$ & $1 / 6$ & $-2 / 3$ & $1 / 3$ & $-1 / 2$ & $-1 / 2$ & $-1 / 2$ & 1 & 1 & 1 \\
$U(1)_{X}$ & 0 & 0 & 0 & $-n$ & 0 & $n$ & $(n-1)$ & 0 & $-(n-1)$ & 0 \\
\hline
\end{tabular}

Table 2 BSM particles and their corresponding charges under complete gauge group

\begin{tabular}{|c|c|c|c|c|c|c|c|c|c|}
\hline \multirow[t]{2}{*}{ Gauge group } & \multicolumn{7}{|c|}{ Fermions } & \multicolumn{2}{|c|}{ Scalars } \\
\hline & $N_{e}^{c}$ & $N_{\mu}^{c}$ & $N_{\tau}^{c}$ & $V L_{l 1}$ & $V L_{l 1}^{c}$ & $V L_{l 3}$ & $V L_{l 3}^{c}$ & $\phi_{1}$ & $\phi_{2}$ \\
\hline $\mathrm{SU}(3)_{c}$ & 1 & 1 & 1 & 1 & 1 & 1 & 1 & 1 & 1 \\
\hline $\mathrm{SU}(2)_{\mathrm{L}}$ & 1 & 1 & 1 & 2 & 2 & 2 & 2 & 1 & 1 \\
\hline$U(1)_{Y}$ & 0 & 0 & 0 & $-1 / 2$ & $1 / 2$ & $-1 / 2$ & $1 / 2$ & 0 & 0 \\
\hline$U(1)_{X}$ & $n$ & 0 & $-n$ & $-(n-1)$ & $(n-1)$ & $(n-1)$ & $-(n-1)$ & 1 & $n$ \\
\hline
\end{tabular}

follows,

$$
\begin{aligned}
& \left(\frac{\partial \mathcal{V}\left(\phi_{h}, \phi_{1}, \phi_{2}\right)}{\partial \phi_{j}}\right)_{v, v_{1}, v_{2}}=0 \\
& \quad \text { and }\left(M_{H}^{2}\right)_{i j}=\left(\frac{\partial^{2} \mathcal{V}\left(\phi_{h}, \phi_{1}, \phi_{2}\right)}{\partial \phi_{i} \partial \phi_{j}}\right)_{v, v_{1}, v_{2}},
\end{aligned}
$$

where $\phi_{i, j}=H, H_{1}, H_{2}$. Since, the neutral part of the Higgs fields are not directly related with the phenomenology in the present work, we neglect further discussion about its diagonalisation and defining the mass eigenstates for the Higgses which are thoroughly studied in the literature, tauon mass, ALP production by the freeze-in mechanism and the axion coupling with the electrons. We have shown that to explain all the phenomenology together we need VLL mass very high and above the reheat temperature. In this work, we use very popular Type-I seesaw mechanism to generate the light neutrino mass in the $\mathrm{eV}$ scale. But this is not the full story because with three right handed neutrinos (which have suitable gauge charge under $U(1)_{X}$ in order to write down the Dirac mass term with the left handed neutrinos) and keeping the interaction terms up to dimension-4, we can not explain the neutrino mass if the oscillation parameters are considered in the $3 \sigma$ range. Therefore, we have introduced

$$
\left(\begin{array}{l}
h \\
h_{1} \\
h_{2}
\end{array}\right)=\left(\begin{array}{ccc}
c_{12}^{\prime} c_{13}^{\prime} & s_{12}^{\prime} c_{13}^{\prime} & s_{13}^{\prime} \\
-s_{12}^{\prime} c_{23}^{\prime}-c_{12}^{\prime} s_{23}^{\prime} s_{13}^{\prime} & c_{12}^{\prime} c_{23}^{\prime}-s_{12}^{\prime} s_{23}^{\prime} s_{13}^{\prime} & s_{23}^{\prime} s_{13}^{\prime} \\
s_{12}^{\prime} s_{23}^{\prime}-c_{12}^{\prime} c_{23}^{\prime} s_{13}^{\prime} & -c_{12}^{\prime} s_{23}^{\prime}-s_{12}^{\prime} c_{23}^{\prime} s_{13}^{\prime} c_{23}^{\prime} c_{13}^{\prime}
\end{array}\right)\left(\begin{array}{l}
H \\
H_{1} \\
H_{2}
\end{array}\right)
$$

where $c_{i j}^{\prime}=\cos \theta_{i j}^{\prime}$ and $s_{i j}^{\prime}=\sin \theta_{i j}^{\prime}$.

As described, in the present model we have extra two complex singlet scalars $\phi_{1}, \phi_{2}$. Among the four components of the extra scalars, two of them are CP even and the other two are $\mathrm{CP}$ odd. One of the $\mathrm{CP}$ odd components becomes the transverse component of the extra gauge boson and the other $\mathrm{CP}$ odd component becomes a stable ALP at the Universe time scale and explains the recent Xenon-1T signal. In most of the previous studies of ALP which explain the Xenon-1T signal is produced thermally and misalignment mechanism. In the present work, we have produced the ALP by the freeze-in mechanism which will be discussed in Sect. 4.1. We also have vector like leptons which are charged in such a way so that the present model is gauge anomaly free. SM and BSM particles are charged under the additional gauge group in such a way so that vector like leptons take an important role in electron, dimension-5 operator in the Dirac mass term of neutrinos and we can successfully explain the neutrino mass with the oscillation parameters in $3 \sigma$ range which will be discussed in Sect. 3. Therefore, to obtain the neutrino mass and mixing angles within the present day accepted bound, we need the dimension-5 operators. Moreover, the only way we can write down the dimension-5 operator with the help of singlet scalar $\phi_{2}$. These dimension-5 operators associated with the right handed neutrino can also produce ALP by the freezein mechanism $\left(N_{i} \rightarrow L_{j} a_{1}\right.$ where $\left.i, j=1,2,3\right)$ but the contribution to the total relic density will be sub dominant because of the Planck scale suppression. Therefore, we have neglected this contribution to our study. But, there are also dimension-4 operators associated with right handed neutri- 
nos and ALP which can produce the ALP through freeze in mechanism by $N_{j} \rightarrow N_{i} a\left(M_{N_{j}}>M_{N_{i}}+m_{a}\right)$ processes. We have shown in detail the production of ALP by the right handed neutrinos decay and considered the relevant constraints. We exhibit the effect of these constraints on the model parameters in Sect. 3. Finally, we can see all the additional particles in the present model are inter-related and we can not explain all the phenomenology together by omitting either of them. We have not discussed much about the additional gauge boson because it does not have a significant role in our phenomenology on which we are interested. Although, this additional gauge boson can be accessed at the colliders when we take tree level kinetic mixing with $U(1)_{Y}$ gauge boson.

\section{Neutrino mass and ALP production}

As given in Eq. (8), we can write down the neutrino mass matrix after the $S U(3)_{c} \times S U(2)_{L} \times U(1)_{Y} \times U(1)_{X}$ gauge groups break down to $S U(3)_{c} \times U(1)_{e m}$ as,

$$
-\mathcal{L}_{N}=\left(\begin{array}{ll}
v & N^{c}
\end{array}\right)\left(\begin{array}{cc}
0 & M_{D}^{T} \\
M_{D} & M_{R}
\end{array}\right)\left(\begin{array}{c}
v \\
N^{c}
\end{array}\right)
$$

where $v=\left(v_{e}, v_{\mu}, v_{\tau}\right), N^{c}=\left(N_{e}^{c}, N_{\mu}^{c}, N_{\tau}^{c}\right)$. The Dirac mass matrix $\left(M_{D}\right)$ and the Majorana mass matrix $\left(M_{R}\right)$ take the following form,

$$
\begin{gathered}
M_{D}=m_{d i j}=\left(\begin{array}{ccc}
\frac{y_{e e} v}{\sqrt{2}} & \frac{y_{e \mu} v v_{2}}{\sqrt{2} M_{P l}} & 0 \\
\frac{y_{\mu e} v v_{2}}{\sqrt{2} M_{P l}} & \frac{y_{\mu \mu} v}{\sqrt{2}} & \frac{y_{\mu \tau} v v_{2}}{\sqrt{2} M_{P l}} \\
0 & \frac{y_{\tau \mu} v v_{2}}{\sqrt{2} M_{P l}} & \frac{y_{\tau \tau} v}{\sqrt{2}}
\end{array}\right), i, j=e, \mu, \tau \\
M_{R}=m_{R i j}=\left(\begin{array}{ccc}
0 & \frac{Y_{e \mu} v_{2}}{\sqrt{2}} & M_{e \tau} e^{i \theta} \\
\frac{Y_{e \mu} v_{2}}{\sqrt{2}} & M_{\mu \mu} & \frac{Y_{\mu \tau} v_{2}}{\sqrt{2}} \\
M_{e \tau} e^{i \theta} & \frac{Y_{\mu \tau} v_{2}}{\sqrt{2}} & 0
\end{array}\right), i, j=e, \mu, \tau .
\end{gathered}
$$

In the case of the Majorana mass matrix, we have considered Yukawa terms up to dimension four whereas for Dirac mass matrix we have considered terms upto dimension five otherwise we do not obtain the neutrino oscillation parameters in the $3 \sigma$ range. In the seesaw approximation after diagonalising the matrix (as shown in Eq. (13)), one can have the light neutrino masses and heavy neutrino masses in the following form,

$$
\begin{aligned}
m_{v} & =-M_{D}^{T} M_{R}^{-1} M_{D} \\
M_{N} & =M_{R} .
\end{aligned}
$$

Matrix diagonalisation (as given in Eq. (13)) in the $\mathcal{O}\left(\frac{m_{D}}{m_{R}}\right)$ happens by the following kind of matrix,

$$
V_{1}=\left(\begin{array}{cc}
-1 & M_{D} M_{R}^{-1} \\
M_{D}^{T} M_{R}^{-1} & 1
\end{array}\right)
$$

In this work, right handed neutrinos not only generate the neutrino masses but also assist in producing the axion like particle (details about the ALP is given in Sect. 4) from its decay. The relevant terms which can produce ALP from the right handed heavy neutrino decay are the terms which consist of right handed neutrinos and one of the extra singlet scalars and are given in Eq. (8). We write down the relevant terms from Eq. (8) which can produce ALP are as follows,

$$
\begin{aligned}
\mathcal{L}_{N}^{A L P}= & y_{e \mu}^{\phi_{2}} L_{e} \phi_{h} N_{\mu}^{c} \frac{\phi_{2}}{M_{P l}}+y_{\mu e}^{\phi_{2}} L_{\mu} \phi_{h} N_{e}^{c} \frac{\phi_{2}^{\dagger}}{M_{P l}} \\
& +y_{\mu \tau}^{\phi_{2}} L_{\mu} \phi_{h} N_{\tau} \frac{\phi_{2}}{M_{P l}}+y_{\tau \mu}^{\phi_{2}} L_{\tau} \phi_{h} N_{\mu}^{c} \frac{\phi_{2}^{\dagger}}{M_{P l}} \\
& +Y_{e \mu} N_{e}^{c} N_{\mu}^{c} \phi_{2}^{\dagger}+Y_{\mu \tau} N_{\mu}^{c} N_{\tau}^{c} \phi_{2}+\text { h.c. }
\end{aligned}
$$

The interaction terms which have Planck scale suppression produce ALP sub-dominantly. Therefore, we focus on the other terms which don't have Planck scale suppression for producing ALP from right handed neutrino decay through freeze in mechanism. In Eq. (18), right handed neutrinos are in interaction basis and we need to write them in mass basis by the following transformation,

$N_{f}=V_{f i} N_{i}, \quad \mathrm{f}=\mathrm{e}, \mu, \tau$ and $\mathrm{i}=1,2,3$

where $V_{f i}$ is an Unitary matrix which diagonalise the matrix $M_{R}$ (given in Eq. (15)). Substituting the flavor basis in terms of the mass basis in Eq. (18) and also considering only the CP odd component of $\phi_{2}$, we get the following kind of interaction terms,

$\mathcal{L}_{N}^{A L P} \supset \kappa_{i j} \bar{N}_{i} P_{R} N_{j} a_{2}+$ h.c. $\quad(i, j=1,2,3)$.

where

$\kappa_{i j}=i\left(-Y_{e \mu} V_{e i}^{\dagger} V_{\mu j}+Y_{\mu \tau} V_{\mu i}^{\dagger} V_{\tau j}\right)$

We can make the transformation of $a_{2}$ and write down it in terms of ALP, $a$, as defined in Eq. (31). We consider $N_{1}$ as the lightest among the three heavy right-handed neutrinos, so we can produce the ALP by the freeze in mechanism from the decay of the other two heavier right handed neutrinos, $N_{2,3}$. In order to produce the ALP by the freeze-in mechanism we have to check the non thermality condition $\frac{\left\langle\Gamma_{N_{j} \rightarrow N_{1} a}\right\rangle}{H}<1$ and we have to solve the following Boltzmann equation in order to calculate the comoving number density of ALP, 


$$
\begin{aligned}
& \frac{d Y_{a}}{d z}=\frac{M_{P l}}{1.66 M_{N_{i}}} \frac{z \sqrt{g_{*}(z)}}{g_{s}(z)} \\
& {\left[\sum_{i=2,3}<\Gamma_{N_{i} \rightarrow N_{1} a}>\left(Y_{N_{i}}^{e q}-Y_{a}\right)\right]}
\end{aligned}
$$

where $Y_{a}$ is the comoving number density of $a, g_{*}(z)$ and $\left(g_{\rho}(z)\right)$ are the matter and entropy d.o.f of the Universe and $\langle\Gamma\rangle$ is the thermal average. The detail description of all the quantities are given in Sect. 4.1. The expression of decay width of $N_{i}, \Gamma_{N_{i} \rightarrow N_{1} a}$, is given in appendix. Once $Y_{a}$ is calculated, we can then determine ALP relic density by the following expression,

$\Omega_{a} h^{2}=2.755 \times 10^{8}\left(\frac{m_{a}}{\mathrm{GeV}}\right) Y_{a}\left(T_{0}\right)$.

We have considered ALP relic density bound in the following range [6],

$0.1172<\Omega_{a} h^{2}<0.1226$.

Moreover, at the time of diagonalising the matrix $M_{R}$ (as given in Eq. (15)) we have used $V_{1}$ matrix (see Eq. (17)) matrix. Therefore, we can relate the heavy right handed neutrino with the light active neutrinos 1.e. we can replace $N_{i}$ by $\left(M_{D} M_{R}^{-1}\right)_{i j} v_{j}$. So, our ALP dark matter can decay to two light neutrinos i.e. $a \rightarrow v_{i} v_{j}(i, j=1,2,3)$. To be a viable DM candidate, ALP life time, $\tau_{A L P}=\frac{1}{\Gamma_{a \rightarrow v_{i} v_{j}}}$, has to be greater than the age of the Universe, $\tau_{U} \sim 5 \times 10^{17} \mathrm{Sec}$. In addition if ALP can decay to photons or to other SM particles which can subsequently produce photons via inverse Compton scattering [88-92] then ALP lifetime is severely constrained by $\gamma$-rays observations. The current experimental bound on ALP decay lifetime can vary from $10^{27}-10^{29}$ sec depending on its mass. In this work, we consider conservative limit on ALP lifetime which is $\tau_{A L P}>10^{27} \mathrm{sec}$. The expression of $\Gamma_{a \rightarrow v_{i} v_{j}}$ is given in the appendix.

To obtain the allowed parameter space, we have considered neutrino oscillation parameters namely sum of the light neutrino masses $\left(\sum_{i=1,2,3} m_{v_{i}}\right)$, two mass square differences $\left(\Delta m_{12}^{2}, \Delta m_{13}^{2}\right)$ and three mixing angles $\left(\theta_{12}, \theta_{13}\right.$ and $\left.\theta_{23}\right)$ in the following range as obtained from Planck collaboration [6] and neutrino oscillation experiments [93],

- cosmological upper bound on the sum of all three light neutrinos, $\sum_{i} m_{i}<0.23 \mathrm{eV}$ at $2 \sigma \mathrm{CL}[6,94,95]$,

- mass squared differences $6.93<\frac{\Delta m_{21}^{2}}{10^{-5}} \mathrm{eV}^{2}<7.97$ and $2.37<\frac{\Delta m_{31}^{2}}{10^{-3}} \mathrm{eV}^{2}<2.63$ in $3 \sigma$ range [93]

- all three mixing angles $30^{\circ}<\theta_{12}<36.51^{\circ}, 37.99^{\circ}<$ $\theta_{23}<51.71^{\circ}$ and $7.82^{\circ}<\theta_{13}<9.02^{\circ}$ also in $3 \sigma$ range [93].
In generating the scatter plots among the model parameters, we have diagonalised the neutrino mass matrices and collected those points which satisfy the neutrino oscillation data as given earlier. We have varied neutrino mass matrix elements in the following range,

$$
\begin{aligned}
& 10^{-8} \mathrm{GeV} \leq m_{d i j} \leq 10^{-4} \mathrm{GeV} \\
& 10^{-6} \mathrm{GeV} \leq m_{R i j} \leq 10^{2} \mathrm{GeV} \\
& 0 \leq \theta \leq \pi
\end{aligned}
$$

where $i, j=\mathrm{e}, \mu, \tau$. Now, we are going to show few scatter plots which are obtained for the normal hierarchy of the neutrino mass matrix and one can obtain similar kind of plots for the inverted hierarchy (IH) of the neutrino masses.

In the LP of Fig. 1, we have shown the variation in the $m_{d e e}-m_{d e \mu}$ plane after satisfying the non thermality (NT) condition of ALP dark matter, constraint on ALP lifetime from the X-ray observation $\left(\tau_{A L P}\right)$, Planck relic density (RD) bound on ALP DM and the $3 \sigma$ bounds on two mass square differences and three mixing angles which come from neutrino oscillation data (NOD). After imposing the constraints of non thermality, X-ray and relic density bounds, one can see that the whole region is allowed. The points which satisfy all the three bounds together represented by the magenta circles. The reason behind the allowance of the whole region is that none of the constraints are solely dependent on the elements of $M_{D}$ matrix. Moreover, we can easily see from the figure that there exist a sharp correlation between the $m_{d e e}$ and $m_{d e \mu}$ parameters once we impose the neutrino oscillation data (NOD) bound and expressed by the green triangle points. They also lie around the same ballpark value. One can see that most of the points satisfy the ratio $\frac{m_{d e \mu}}{m_{d e e}} \sim 0.6$ and from oscillation experiments we also know that $\tan \left(\theta_{12}\right) \sim 0.6$. So we can conclude that this type of sharp correlation is coming from the $\theta_{12}$ bound of the oscillation experiment. There are other points also on the upper side due to the variation of the other parameters. On the other hand in the RP of the figure, we have shown the variation in the $m_{d e e}-m_{R e \tau}$ plane after satisfying the non thermality, X-ray bound, relic density and neutrino oscillation data. Cyanide circle points are obtained after demanding the non thermality condition, brick circle hole points are obtained after demanding ALP decay lifetime greater than $\sim 10^{27} \mathrm{sec}$ which comes from $\mathrm{X}$-ray observation, magenta circle points are obtained when we impose the DM relic density constraint and the green triangle points are obtained after imposing the neutrino oscillation data bound. In the case of non thermality constraint whole region is allowed because neither of the parameters is directly related to this condition. In the case of decay lifetime of ALP DM, we can see that higher values of $M_{R e \tau}$ are allowed because the coupling of ALP decay to two neutrinos is inversely proportional to the square of the elements of $M_{R}$. So, higher values of $M_{R} e \tau$ help in getting the larger value of 


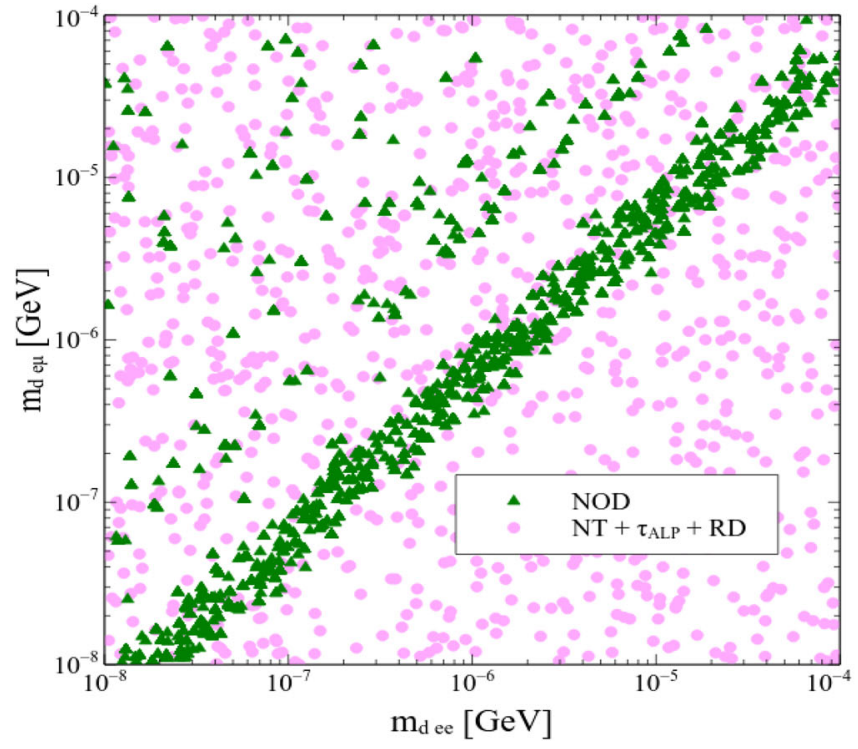

Fig. 1 LP (RP): Scatter plot in the $m_{d e e}-m_{d e \mu}\left(m_{d e e}-m_{R e \tau}\right)$ plane after satisfying the non thermality (NT) condition of ALP, ALP lifetime which is greater than the age of the $\operatorname{Universe}\left(\tau_{U}\right)$, Planck relic den-

the ALP decay lifetime to become a viable DM candidate by satisfying the constraint from X-ray observation. Moreover, in the case of relic density constraint higher values of $M_{R e \tau}$ gives the correct value of relic density obtained from Planck data. Finally, when we apply the NOD bound, we notice that there exist a correlation between these two parameters. This correlation is trying to obey the bound $\frac{m_{\text {dee }}^{2}}{m_{\text {Ret }}} \leq 10^{-10} \mathrm{GeV}$ which is coming from the two mass square differences $\Delta m_{12}^{2}$, $\Delta m_{13}^{2}$ and sum of neutrino masses $\sum_{i=1,2,3} m_{v_{i}}$. There is also upper bound in the same plane for lower values of $m_{d e e}$ which is just coming from the bound $\frac{m_{d e e}^{2}}{m_{R e \tau}} \geq 10^{-15}$ because below this value mass the square differences are very difficult to obtain in the $3 \sigma$ range. We can also notice that the region at top right corner satisfies all the constraints together i.e. the overlapping region between magenta and green points.

Figure 2 shows the variation in the $m_{d e \mu}-m_{R e \tau}$ and $m_{R \mu \mu}-m_{R \mu \tau}$ planes after satisfying all the constraints we have imposed in Fig. 1. The colour coding has the same meaning as we have for Fig. 1. In particular, the LP draws the same kind of physics conclusion as explained for the RP of Fig. 1. Here also when we apply NOD the lower bound is coming from constraint $\frac{m_{d e \mu}^{2}}{m_{R e \tau}} \leq 10^{-10}$ and a sharp upper bound is coming from $\frac{m_{d e \mu}^{2}}{m_{R e \tau}} \geq 10^{-14}$. In the RP we have shown the variation in $m_{R \mu \mu}-m_{R \mu \tau}$ plane after satisfying all the bounds. In particular, we can see that when we impose the relic density and X-ray bounds both the parameters can not take lower value together either of them have to have a larger value. Otherwise, it increases $M_{D} / M_{R}$ ratio

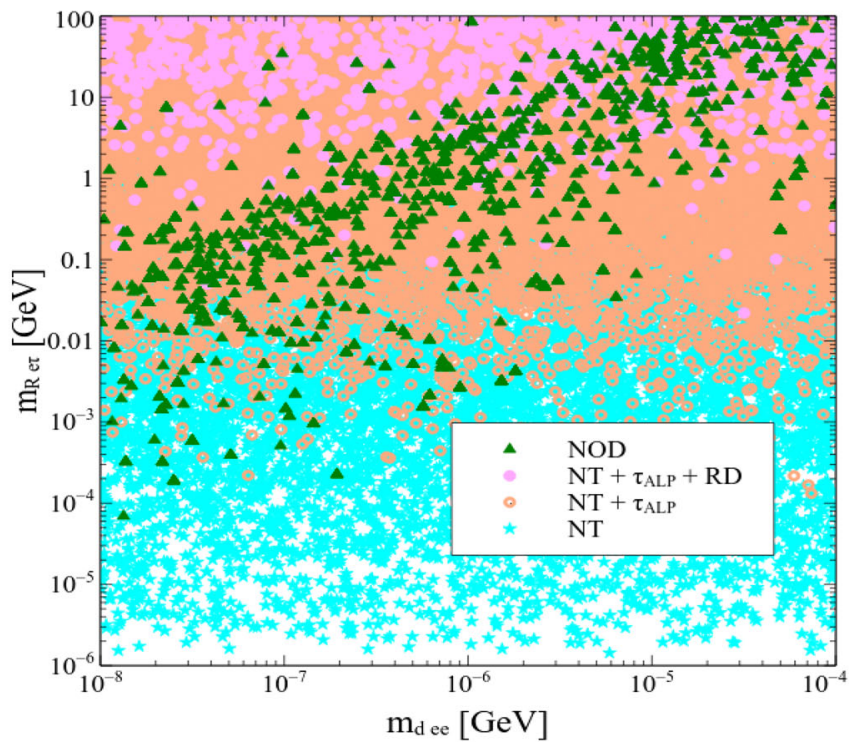

sity (RD) bound of ALP and the bound from neutrino oscillation data (NOD). Other parameters have been kept fixed at $v_{1,2}=10^{10} \mathrm{GeV}$ and $n=10$

which in turn increase the coupling strength and decrease the lifetime of ALP dark matter. We can also see a correlation among the magenta points for the higher value of $m_{R \mu \mu}$. This is because DM relic density is proportional to the square of $m_{R \mu \tau}\left(=\frac{Y_{\mu \tau} v_{2}}{\sqrt{2}}\right)$ whereas it is inversely proportional to the right handed neutrino masses (which are linearly proportional to the elements of $M_{R}$ ). Therefore, to get the correct value of the relic density, these two parameters try to have correlated values. Moreover, when we impose NOD constraint we can see from the figure that both the parameter can not take low values simultaneously but they can take higher values together. Furthermore, either of them can take a low value but at the same time, the other parameter has to take a high value to satisfy the neutrino oscillation data.

\section{Xenon-1T signal}

As said in the model part (Sect. 2), the CP even parts of the scalar fields are not directly related with the phenomenology we are interested in this work. On the other hand, the CP odd part of the singlet Higgses take a pivotal role in the present work, hence we are going to discuss now the $\mathrm{CP}$ odd components. $\mathrm{CP}$ odd part can be written in the following manner,

$\phi_{1}=\frac{v_{1}}{\sqrt{2}} e^{i \frac{a_{1}}{v_{1}}}$ and $\phi_{2}=\frac{v_{2}}{\sqrt{2}} e^{i \frac{a_{2}}{v_{2}}}$

where it follows the vev condition $\left|\phi_{i}\right|^{2}=\frac{v_{i}^{2}}{2}$. Kinetic term for the extra singlet scalars and $\left(U(1)_{X}\right)$ gauge boson take 


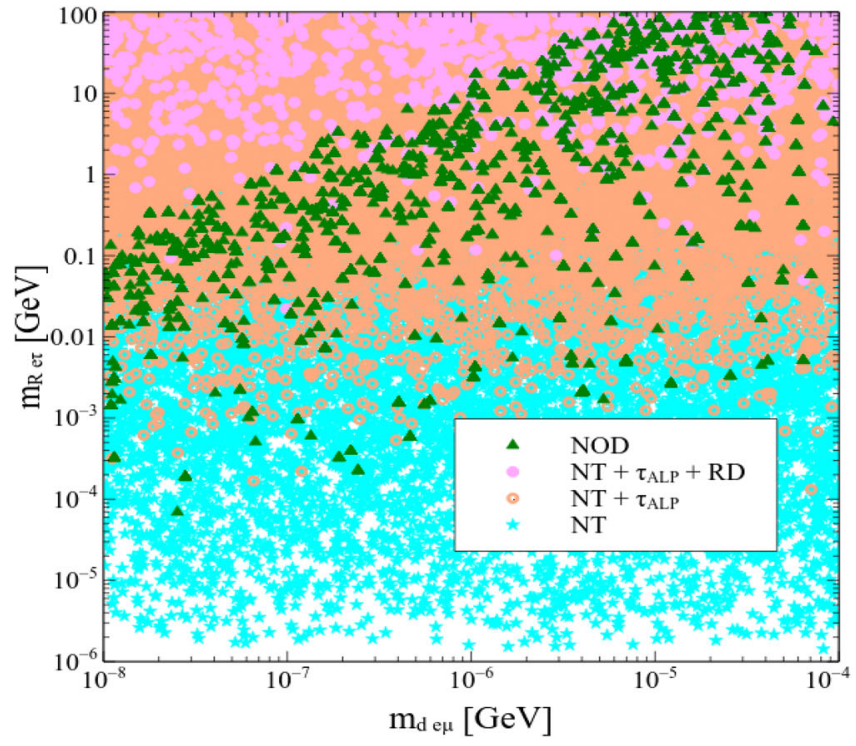

Fig. 2 LP (RP): Scatter plot in the $m_{d e \mu}-m_{R e \tau}\left(m_{R \mu \mu}-m_{R \mu \tau}\right)$ plane after satisfying the non thermality (NT) condition of ALP, ALP lifetime greater than the age of the Universe $\left(\tau_{U}\right)$, Planck relic den-

the following form,

$\mathcal{L}_{\text {kin }}^{\phi_{i}}=-\frac{1}{4} F_{\mu \nu}^{\prime} F^{\prime \mu \nu}+\sum_{i=1,2}\left|D_{\mu} \phi_{i}\right|^{2}$

where $F_{\mu \nu}^{\prime}$ is the field strength tensor associated with $U(1)_{X}$ gauge group. The covariant derivative takes the following form,

$D_{\mu}^{i}=\partial_{\mu}-i g^{\prime} Z^{\prime} n_{i}$

where $n_{i}$ and $g^{\prime}$ are the $U(1)_{X}$ gauge charge and gauge coupling. By expanding the covariant derivative in Eq. (27), we have the kinetic term as follows,

$$
\begin{aligned}
\mathcal{L}_{\text {kin }}^{\phi_{i}}= & -\frac{1}{4}\left(F_{\mu \nu}^{\prime}\right)^{2}+\frac{1}{2}\left(\partial_{\mu} a_{1}\right)^{2}+\frac{1}{2}\left(\partial_{\mu} a_{2}\right)^{2} \\
& +\frac{M_{Z^{\prime}}^{2}}{2}\left(Z_{\mu}^{\prime}\right)^{2}-g^{\prime} Z_{\mu}^{\prime} \partial^{\mu}\left[n_{1} v_{1} a_{1}+n_{2} v_{2} a_{2}\right]
\end{aligned}
$$

where the gauge boson mass has the following form,

$M_{Z^{\prime}}=g^{\prime 2}\left(n_{1}^{2} v_{1}^{2}+n_{2}^{2} v_{2}^{2}\right)$.

We can redefine $a_{1}$ and $a_{2}$ in terms of mass basis $a$ and $G_{Z^{\prime}}$ as follows,

$$
\begin{aligned}
a & =\frac{1}{\sqrt{n_{1}^{2} v_{1}^{2}+n_{2}^{2} v_{2}^{2}}}\left[n_{2} v_{2} a_{1}-n_{1} v_{1} a_{2}\right] \\
G_{Z^{\prime}} & =\frac{1}{\sqrt{n_{1}^{2} v_{1}^{2}+n_{2}^{2} v_{2}^{2}}}\left[n_{1} v_{1} a_{1}+n_{2} v_{2} a_{2}\right] .
\end{aligned}
$$

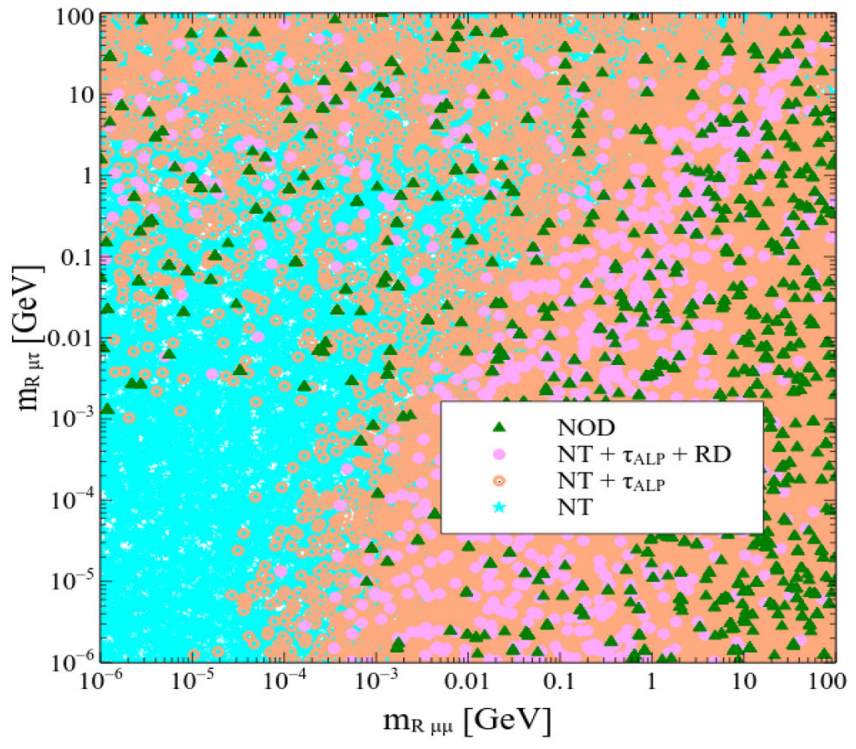

sity (RD) bound of ALP and the bound from neutrino oscillation data (NOD). Other parameters have been kept fixed at $v_{1,2}=10^{10} \mathrm{GeV}$ and $n=10$

Therefore, in terms of $a$ and $G_{Z^{\prime}}$ Eq. (29) takes the following form,

$$
\begin{aligned}
\mathcal{L}_{\text {kin }}^{\phi_{i}}= & -\frac{1}{4}\left(F_{\mu \nu}^{\prime}\right)^{2}+\frac{1}{2}\left(\partial_{\mu} a\right)^{2} \\
& +\frac{1}{2} M_{Z^{\prime}}^{2}\left(Z^{\prime}-\frac{\partial_{\mu} G_{Z^{\prime}}}{M_{Z^{\prime}}}\right)^{2} .
\end{aligned}
$$

The above Lagrangian is in Stückelberg form and it is clearly visible that the $G_{Z^{\prime}}$ degree of freedom is becoming the transverse component of $Z^{\prime}$ and makes it massive. The other $\mathrm{CP}$ odd component " $a$ " is massless. If we consider the higher dimensional operator which is consist of $\phi_{1}$ and $\phi_{2}$ as given in Eq. (9),

$\mathcal{V}^{H D O}=\frac{\lambda_{\phi_{1} \phi_{2}} \phi_{1}^{n} \phi_{2}^{\dagger}}{M_{P l}^{n-3}}+$ h.c.

By using the form as given in Eq. (26), we can write the above $\mathrm{HDO}$ in the following way,

$$
\begin{aligned}
\mathcal{V}^{H D O} & =\frac{\lambda_{\phi_{1} \phi_{2}} v_{1}^{n} v_{2}}{2^{\frac{n+1}{2}} M_{P l}^{n-3}} e^{i\left(\frac{n v_{2} a_{1}-v_{1} a_{2}}{v_{1} v_{2}}\right)}+\text { h.c. } \\
& =\frac{\lambda_{\phi_{1} \phi_{2}} v_{1}^{n} v_{2}}{2^{\frac{n+1}{2}} M_{P l}^{n-3}} e^{i \frac{a}{V}}+\text { h.c. }
\end{aligned}
$$

where $V=\frac{v_{1} v_{2}}{\sqrt{n_{1}^{2} v_{1}^{2}+n_{2}^{2} v_{2}^{2}}}$ and we have used the definition of $a$. Expanding the above expression we get,

$\mathcal{V}^{H D O}=\frac{2 \lambda_{\phi_{1} \phi_{2}} v_{1}^{n} v_{2}}{2^{\frac{n+1}{2}} M_{P l}^{n-3}}\left(1-\frac{a^{2}}{2 V^{2}}+\cdots\right)$. 


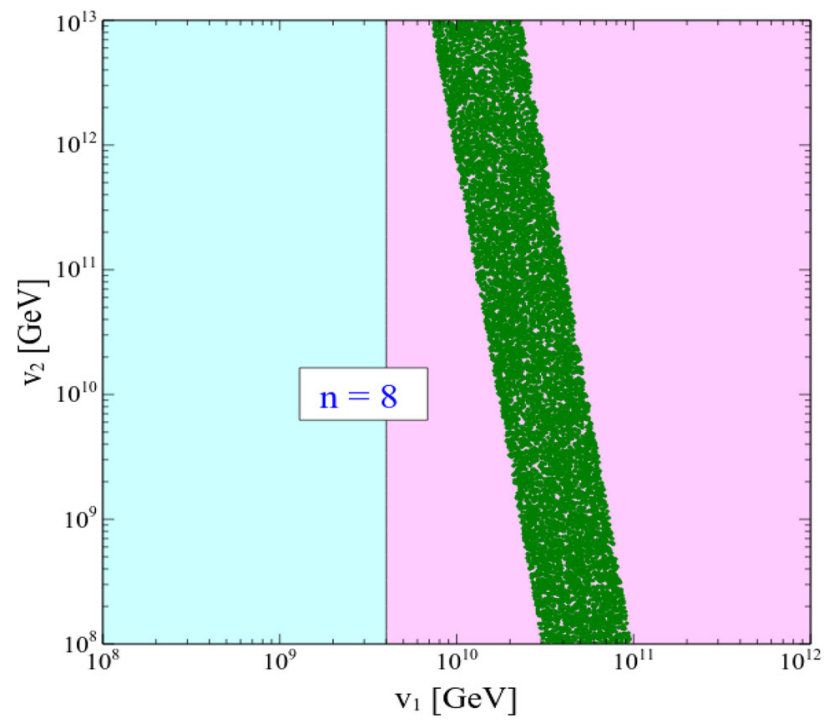

Fig. 3 Scatter plot in the $v_{1}-v_{2}$ plane where green points are after demanding ALP mass in between $1-100 \mathrm{keV}$. Magenta region satisfies the electron and tauon mass by suitably choosing $y_{e}, y_{1}^{V L}, y_{3}^{V L}$ and $y_{\tau}$ values. Cyanide region is disallowed by the electron and tauon mass. We have kept fixed $\lambda_{\phi_{1} \phi_{2}}=1, y_{1,3}^{\phi_{1}}=10^{-8}$ and considered reduced value of the Planck mass, $M_{P l}=2.43 \times 10^{18} \mathrm{GeV}$

Finally we get mass of ALP $a$ which is $m_{a}^{2}=\frac{2 \lambda_{\phi_{1} \phi_{2}} v_{1}^{n} v_{2}}{2^{\frac{n+1}{2}} M_{P l}^{n-3} V^{2}}$.

In Fig. 3, we have shown the variation in the $v_{1}-v_{2}$ plane for $n=8$ which is the $U(1)_{X}$ charge of $\phi_{2}$. Magenta region is allowed from the electron and tauon mass whereas cyanide region is disallowed from the electron and muon mass. Green points are obtained after demanding the ALP mass in the 1$100 \mathrm{keV}$ range. To satisfy, the electron mass, muon mass and axion mass in the $\mathrm{keV}$ range, we need $n \geq 8$ which is the $U(1)_{X}$ charge of $\phi_{2}$ singlet scalar. We can say from the electron, muon and $\mathrm{keV}$ order ALP mass bound that the $U(1)_{X}$ charge of $n<8$ is already ruled out. Although, in generating the scatter plot we have considered $\lambda_{\phi_{1} \phi_{2}}=1$ and if we take the other value of $\lambda_{\phi_{1} \phi_{2}}$ then it will accordingly change the bound on $n$.

Since our main target is to explain the Xenon-1T signal, we have to discuss about the ALP coupling with electrons. As given in Eq. (7) if we integrated out the vector like leptons (more discussion is in Sect. 4.1.2) then we get the following terms,

$$
\begin{aligned}
& \mathcal{L}_{\text {lepton }} \supset-\frac{y_{1}^{V L} y_{1}^{\phi_{1}}}{M_{1}^{V L}} \phi_{1} L_{e} E_{e}^{c} \phi_{h}-y_{e} L_{e} \phi_{h} E_{e}^{c} \frac{\phi_{1}}{M_{P l}} \\
& -y_{\mu} L_{\mu} \phi_{h} E_{\mu}^{c}-\frac{y_{3}^{V L} y_{3}^{\phi_{1}}}{M_{3}^{V L}} \bar{\phi}_{1} L_{\tau} E_{\tau}^{c} \phi_{h} \\
& -y_{\tau} L_{\tau} \phi_{h} E_{\tau}^{c} \frac{\overline{\phi_{1}}}{M_{P l}}+\text { h.c. }
\end{aligned}
$$

once $\phi_{1}$ and $\phi_{h}$ take vevs then we get,

$\mathcal{L}_{\text {lepton }} \supset-m_{e} l_{e} E_{e}^{c} e^{i \frac{a_{1}}{v_{1}}}-m_{\mu} l_{\mu} E_{\mu}^{c}-m_{\tau} l_{\tau} E_{\tau}^{c} e^{-i \frac{a_{1}}{v_{1}}}+$ h.c.

where $m_{e}=\left(\frac{y_{1}^{V L} y_{1}^{\phi_{1}} v v_{1}}{2 M_{1}^{V L}}+\frac{y_{e} v v_{1}}{2 M_{P l}}\right), m_{\mu}=\frac{y_{\mu} v}{\sqrt{2}}$ and $m_{\tau}=$ $\left(\frac{y_{3}^{V L} y_{3}^{\phi_{1}} v v_{1}}{2 M_{3}^{V L}}+\frac{y_{\tau} v v_{1}}{2 M_{P l}}\right)$ are the electron, muon and tauon mass. One important think to note here is that the ALP coupling to electron $\left(g_{a e e}\right)$ is given by

$g_{a e e} \simeq \frac{m_{e} V}{v_{1}^{2}}=2.5 \times 10^{-14}\left(\frac{V \times 2 \times 10^{10} \mathrm{GeV}}{v_{1}^{2}}\right)$.

We consider a global symmetry $U(1)_{g}$ and leptons are charged under this global symmetry. Once the global symmetry gets broken then pseudo Nambu Goldstone boson (PNGB) is produced which acquire keV order mass from the higher dimensional operator as discussed before. We assign the global charge in such a way so that it is anomaly free and naturally the decay of PNGB to $\gamma \gamma$ is suppressed. We are working in $\mathrm{keV}$ range $\mathrm{PNGB}$, hence it will not decay directly to electrons but can decay to $\gamma \gamma$. By integrating out electron and tauon, we generate the following kind of interaction [96],

$$
\begin{aligned}
& \mathcal{L}_{e f f} \simeq-(1-1) \frac{\alpha_{e m}}{4 \pi v_{1}} a_{1} F_{\mu \nu} \tilde{F}^{\mu \nu} \\
& +\frac{\alpha_{e m}}{48 \pi v_{1}}\left(\frac{1}{m_{e}^{2}}-\frac{1}{m_{\tau}^{2}}\right)\left[\left(\partial^{2} a_{1}\right) F_{\mu \nu} \tilde{F}^{\mu \nu}+2 a_{1} F_{\mu \nu} \partial^{2} \tilde{F}^{\mu \nu}\right],
\end{aligned}
$$

first term comes from the anomaly $U(1)_{g}-U(1)_{e m}-U(1)_{e m}$ and we chose the global charge such that the anomaly is zero in the present case. The second term is the threshold correction. This threshold correction is suppressed by the axion and electron mass ratio $\left(\frac{m_{a}}{m_{e}}\right)^{2}$ which is around $10^{-5}$ for keV scale axion. Because of this suppression, the ALP dark matter in the present model is safe from the astrophysical and cosmological constraints mainly the X-ray bound. Moreover, if the ALP is produced in the star then it is stringently restricted from the stellar cooling bound mainly from red giants and white dwarfs. In principle, if ALP is $\gamma$-philic then we can overcome the bound because $g_{a \gamma \gamma}$ is very much suppressed in the present model due to the non-anomalous global symmetry. On the other hand, if ALP is $\gamma$-phobic then we need to suppressed the $g_{a e e}$ coupling and one such example is chameleon type ALP dark matter which has been studied in [28,97-103]. Chameleon type ALP dark matter production is suppressed at the high density region and it is unaltered at the low density region. Therefore, ALP dark matter production at very dense stellar objects like red giants and white dwarfs is very much suppressed whereas its production in less dense objects like the sun remains unchanged. Since we will be dealing with the on shell PNGB, hence we can use 
the on shell condition which turn the above equation into the following form,

$\mathcal{L}_{e f f} \simeq \frac{\alpha_{e m} V m_{a}^{2}}{48 \pi v_{1}^{2}}\left(\frac{1}{m_{e}^{2}}-\frac{1}{m_{\tau}^{2}}\right) a F_{\mu \nu} \tilde{F}^{\mu \nu}$

where we have used $a_{1}=\frac{n_{1} v_{1} G_{Z^{\prime}}+n_{2} v_{2} a}{\sqrt{n_{1}^{2} v_{1}^{2}+n_{2}^{2} v_{2}^{2}}}$. Decay rate of PNGB, $a$, to $\gamma \gamma$ is given by,

$\Gamma_{a \rightarrow \gamma \gamma} \simeq \frac{\alpha_{e m} m_{a}^{7}}{9216 \pi^{3}\left(\frac{v_{1}^{2}}{V}\right)^{2}} \times \frac{1}{m_{e}^{4}}$.

We can now estimate the lifetime of the $\mathrm{keV}$ scale axion like particle a which only decay to $\gamma \gamma$ (since $m_{a}<2 m_{l}, m_{l}$ is the lepton mass) is given by,

$$
\begin{aligned}
\tau_{a \rightarrow \gamma \gamma}= & \frac{1}{\Gamma_{a \rightarrow \gamma \gamma}} \simeq 3.9 \times 10^{31}\left(\frac{m_{a}}{2.5 \mathrm{keV}}\right)^{-7} \\
& \times\left(\frac{v_{1}^{2}}{V \times 10^{10} \mathrm{GeV}}\right)^{2} .
\end{aligned}
$$

The decay lifetime of the ALP is larger than the age of the Universe and also safe from the X-ray bound, hence it can be a viable DM candidate. In the literature this kind of DM has been produced thermally and misalignment mechanism as described in $[56,96]$. By suitably choosing the ALP decay constant, one can reduce the thermal production of ALP. Moreover, We assume that inflation happens after the global symmetry gets broken. Therefore, we can reduce the DM production by misalignment mechanism by choosing the lower value of the initial oscillation amplitude. In the present work, we are going to produce the $\mathrm{keV}$ scale DM by the freeze-in mechanism namely from the decay of the vector like fermion and from the HDO as given in Eqs. (7), (36). Now we are going to discuss the freeze-in production of $\mathrm{keV}$ scale ALP by two different kind of interactions.

\section{$4.1 \mathrm{keV}$ scale FIMP DM}

\subsubsection{Decay contribution}

As given in Eq. (7), we can produce the $\mathrm{keV}$ scale DM from the decay of the vector like doublet fermion through freezein mechanism by making the corresponding coupling in the feeble range. In Fig. 4, we have shown the out of equilibrium condition of $\mathrm{keV}$ range $\mathrm{DM}$ for $5 \mathrm{TeV}$ vector like lepton for three different values of coupling strength.

In Fig. 4, we have shown the variation of $\frac{\langle\Gamma\rangle}{H}$ with $z$ where $\langle\Gamma\rangle=\Gamma_{V L_{l 1} \rightarrow L a} \frac{K_{1}(z)}{K_{2}(z)}$ and $H=\frac{1.66 \sqrt{g_{*}^{\rho}} T^{2}}{M_{P l}}$ are the thermal average of the decay width and Hubble parameter, respectively. $K_{1}(z), K_{2}(z)$ are the modified Bessel function for the first kind and second kind, $g_{*}^{\rho}$ is the matter d.o.f of the Universe and $z=\frac{M_{V L_{l 1}}}{T}$. In the figure one can easily see that

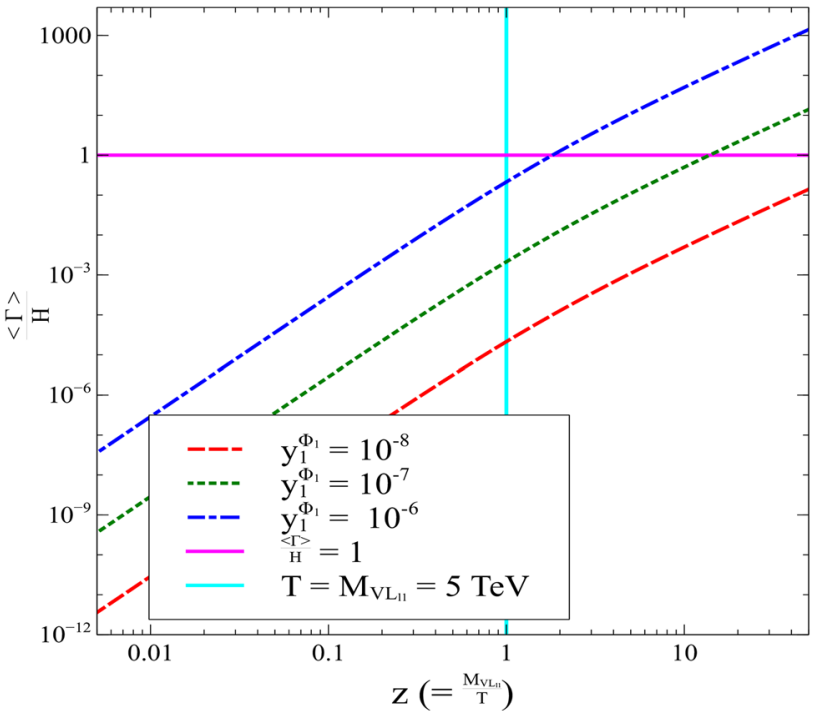

Fig. $4 \frac{\langle\Gamma\rangle}{H}$ variation with $\mathrm{z}$ for three different values of $y_{1}^{\phi_{1}}$

at $T \sim M_{V L_{l 1}}$, the out of equilibrium condition $\frac{\langle\Gamma\rangle}{H}<1$ is always satisfied. If we increase the $y_{1}^{\phi_{1}}$ value greater than $10^{-6}$, then we say that it will reach equilibrium. So to be on the safe side we consider in the present work $y_{1,3}^{\phi_{1}} \sim 10^{-8}$ and the vector like lepton mass in the $\mathrm{TeV}$ range. The Boltzmann equation for determining the decay contribution takes the following form,

$\frac{d Y_{a}}{d z}=\frac{M_{P l}}{1.66 M_{V L_{l 1}}} \frac{z \sqrt{g_{*}(z)}}{g_{s}(z)}\left[\sum_{i=1,3}\left\langle\Gamma_{V L_{l i} \rightarrow L a}\right\rangle\left(Y_{V L_{l i}}^{e q}-Y_{a}\right)\right]$

where $Y_{a}=\frac{n_{a}}{s}$ is the comoving number density of a and $\mathrm{s}$ is the entropy, $s=\frac{2 \pi^{2} g_{*}^{s} T^{3}}{45}$, of the Universe. $g_{*}(z)$ is a parameter which depends on the matter $\left(g_{\rho}(z)\right)$ and entropy $\left(g_{s}(z)\right)$ d.o.f of the Universe in the following way,

$\sqrt{g_{*}(z)}=\frac{g_{s}(z)}{\sqrt{g_{\rho}(z)}}\left(1-\frac{1}{3} \frac{d \ln g_{s}(z)}{d \ln z}\right)$.

$\left\langle\Gamma_{V L_{l i} \rightarrow L a}\right\rangle$ is the thermal average of the decay width of vector like lepton $V L_{l i}(\mathrm{i}=1,3)$ as defined earlier. As shown in $[82,83]$, one can approximately solve the above Boltzmann equation and gets the following analytical expression of the DM relic density,

$\Omega_{a} h^{2}=\sum_{i=1,3} \frac{1.09 \times 10^{27} g_{V L_{l i}} m_{a} \Gamma_{V L_{l i}}}{g_{*}^{s} \sqrt{g_{*}^{\rho}} M_{V L_{l i}}^{2}}$.

In generating the Fig. 5, we have varied the three parameters namely ALP mass $m_{a}$, Yukawa coupling $y_{3}^{\phi_{1}}$ and vector like lepton mass $M_{V L_{l 3}}$ in the following range, ${ }^{1}$

\footnotetext{
${ }^{1}$ We have also assumed that same kind of contribution comes for $V L_{l 1}$ decays and has been taken into account in the decay contribution.
} 

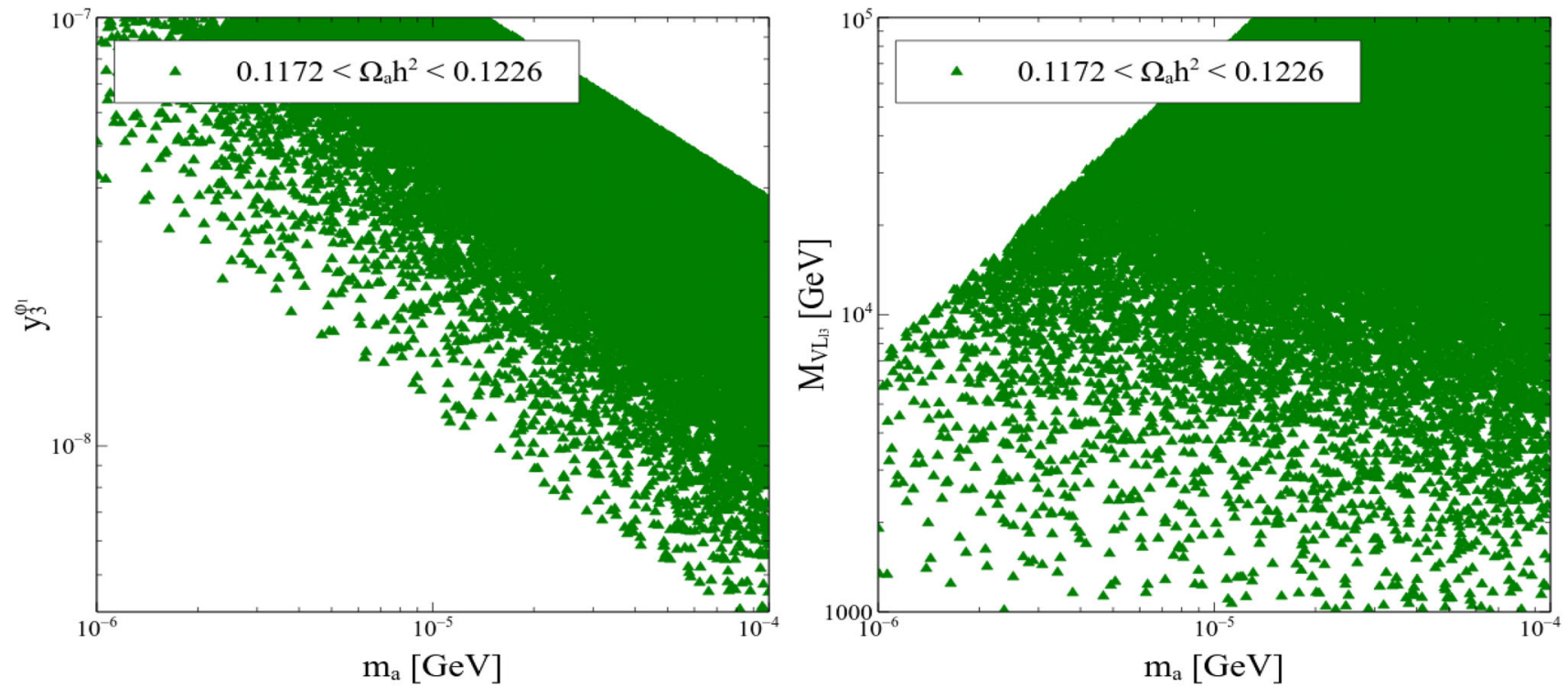

Fig. 5 LP (RP): Scatter plot in $m_{a}-y_{3}^{\phi_{1}}\left(m_{a}-M_{V L_{l 3}}\right)$ plane after satisfying the DM relic density bound obtained from Planck satellite

$$
\begin{aligned}
& 1 \mathrm{keV} \leq m_{a} \leq 100 \mathrm{keV} \\
& 10^{-7} \leq y_{3}^{\phi_{1}} \leq 10^{-10} \\
& 1 \mathrm{TeV} \leq M_{V L_{l 3}} \leq 100 \mathrm{TeV}
\end{aligned}
$$

and we have used Eq. (45) for calculating the DM relic density. In the LP of Fig. 5, we have shown the variation of dark matter mass with the coupling $y_{3}^{\phi_{1}}$. Here, we have considered vector like lepton $V L_{l 3}$ decay to dark matter. All the points satisfy the DM relic density bound as obtained by the Planck collaboration [6]. As given in Eq. 45, the decay width takes the form, when the daughter particles have negligible mass in compared to mother particle,

$\Gamma_{V L_{l 3}}=\frac{\left(y_{3}^{\phi_{1}}\right)^{2}}{16 \pi} M_{V L_{l 3}}$.

We can now easily see from Eq. (45) that relic density is proportional to the DM mass $m_{a}$ and the square of the Yukawa coupling $y_{3}^{\phi_{1}}$ i.e. $\Omega_{a} h^{2} \propto m_{a}\left(y_{3}^{\phi_{1}}\right)^{2}$. To satisfy the DM relic density it is clear that both of them can not increase or decrease simultaneously, in other words, there must exist anti-correlation between $m_{a}$ and $y_{3}^{\phi_{1}}$ which is visible in the LP of the figure. One can also notice that there is also a disallowed region in the upper corner of the figure which corresponds to the higher value of DM mass and large Yukawa coupling. This region overproduces the DM hence is ruled out by the relic density bound. On the other hand in the RP of the figure, we have shown variation in the $m_{a}-M_{V L_{l 3}}$ plane after satisfying the DM relic density. As can be seen from the Eq. (45) the DM relic density varies as $\Omega_{a} h^{2} \propto \frac{m_{a}}{M_{V L_{l 3}}}$. Therefore, to satisfy the DM relic density bound from Planck we expect that both of them will either increase or decrease at the same time which means we expect a sharp correlation among the parameters which is clearly shown in the RP of the figure.

\subsubsection{Contribution from higher dimensional operator}

Once we get rid of the vector type lepton $V L_{L 1}, V L_{L 3}$, then we get the following dimension-5 operator,

$$
\begin{aligned}
\mathcal{L}_{\text {dim }-5} & =\frac{y_{1}^{V L} y_{1}^{\phi_{1}}}{M_{V L_{l 1}}} \phi_{1} L_{e} E_{e}^{c} \phi_{h}+\frac{y_{3}^{V L} y_{3}^{\phi_{1}}}{M_{V L_{l 3}}} \bar{\phi}_{1} L_{\tau} E_{\tau}^{c} \phi_{h}+\text { h.c. } \\
& =\lambda_{1} \phi_{1} L_{e} E_{e}^{c} \phi_{h}+\lambda_{3} \bar{\phi}_{1} L_{\tau} E_{\tau}^{c} \phi_{h}+\text { h.c. }
\end{aligned}
$$

The coupling strength of the above interactions are $\lambda_{i}=$ $\frac{y_{i}^{V L} y_{i}^{\phi_{1}}}{M_{V L_{l i}}} \sim 10^{-11}(\mathrm{i}=1,3)$ which is in the ballpark value of the freeze-in coupling strength. ${ }^{2}$ As discussed in [83], we can apply the freeze-in mechanism to produce the DM from the above non-renormalisable interaction terms. As discussed for decay contribution, vector like lepton is in thermal equilibrium and ALP is produced from its decay. In this case to achieve tauon mass $\left(m_{\tau}\right)$ we need $v_{1} \sim 10^{16} \mathrm{GeV}$ (where tauon mass, $m_{\tau}=\frac{y_{\tau} v v_{1}}{M_{P l}}$ ) but this higher value of the vev can not explain the Xenon-1T signal because it significantly reduces the ALP coupling strength to electrons as given in Eq. (38). Therefore, to get the electron and tauon mass and the Xenon-1T benchmark point, we have to consider the higher dimensional operator (HDO) as given in Eq. (48). Since the vector like lepton is integrated out, so in this case the vector like lepton mass $\left(M_{V L_{l i}}, \mathrm{i}=1,3\right)$ has to be higher than the reheat temperature of the Universe. So, for this scenario

\footnotetext{
$2 \lambda_{1}$ is suppressed by the electron mass so in our case $\lambda_{3}$ takes an important role in DM production.
} 
when $M_{V L_{l i}}>T_{R}(\mathrm{i}=1,3)$, the ALP is not produced from the decay of $V L_{l i}(i=1,3)$ but it is produced from the HDO as given in Eq. (48). For this case, the coupling $\lambda_{3}$ is suppressed by the higher mass value of the vector like lepton and we can choose $y_{3}^{V L}, y_{3}^{\phi_{1}} \sim \mathcal{O}(1)$.

In the present case, we will be considering higher values of vector like lepton mass and the reheat temperature (although $M_{V L_{l 3}}>T_{R}$ ) which implies that UV contribution is more relevant to us than the IR contribution. Therefore, we will be focusing on the contribution coming from the non-renormalisable operator (as shown in Eq. (36)) which depends on the unknown UV physics like reheat temperature $T_{R}$. Considering the non-renormalisable operator only, we can write down the Boltzmann equation for the production of the ALP $a$ as follows [83],

$$
\begin{aligned}
& \frac{d n_{a}}{d t}+3 n_{a} H \simeq \int d \pi_{a} d \phi_{h} d \pi_{L_{\tau}} d_{E_{\tau}^{c}}(2 \pi)^{4} \delta^{4}\left(p_{L_{\tau}}\right. \\
& \left.\quad+p_{E_{\tau}^{c}}-p_{a}-p_{\phi_{h}}\right)|M|_{L_{\tau} E_{\tau}^{c} \rightarrow a \phi_{h}}^{2} f_{L_{\tau}} f_{E_{\tau}^{c}} .
\end{aligned}
$$

After manipulating the above equation we get,

$$
\begin{aligned}
& \frac{d n_{a}}{d t}+3 n_{a} H \simeq \frac{T}{2048 \pi^{6}} \\
& \int d s d \Omega \sqrt{s}|M|_{L_{\tau} E_{\tau}^{c} \rightarrow a \phi_{h}}^{2} K_{1}\left(\frac{\sqrt{s}}{T}\right),
\end{aligned}
$$

where $s$ is the center of mass energy of the $2 \rightarrow 2$ process. Considering the fact that masses of the interacting particles are negligible compared to the temperature we are working on. In this limit, the matrix element is expressed as $|M|_{L_{\tau} E_{\tau}^{c} \rightarrow a \phi_{h}}^{2}=\lambda_{3}^{2} s$. After using this expression Eq. (50) takes the following form,

$\frac{d n_{a}}{d t}+3 n_{a} H \simeq \frac{T \lambda_{3}^{2}}{512 \pi^{5}} \int_{0}^{\infty} d s s^{3 / 2} K_{1}\left(\frac{\sqrt{s}}{T}\right)$.

Defining the comoving number density, $Y_{U V}=\frac{n_{a}}{s}$ and after integration, we get

$\frac{d Y_{U V}}{d T} \simeq-\frac{1}{s H T} \frac{T^{6} \lambda_{3}^{2}}{16 \pi^{5}}$.

Now, using the expression of entropy (s) and Hubble parameter $(\mathrm{H})$, we get from the above equation after integration,

$Y_{U V} \simeq \frac{0.4 T_{R} \lambda_{3}^{2} M_{P l}}{\pi^{7} g_{*}^{s} \sqrt{g_{*}^{\rho}}}$.

Therefore, the relic density would be,

$\Omega_{a}^{U V} h^{2} \simeq 2.755 \times 10^{2}\left(\frac{m_{a}}{\mathrm{keV}}\right) Y_{U V}$.

In Fig. 6, we have shown the allowed region in the $\lambda_{3}-T_{R}$ plane where $\lambda_{3}=\frac{y_{3}^{V L} y_{3}^{\phi_{1}}}{M_{V L_{l 3}}}$ and $T_{R}$ is thereheat temperature.

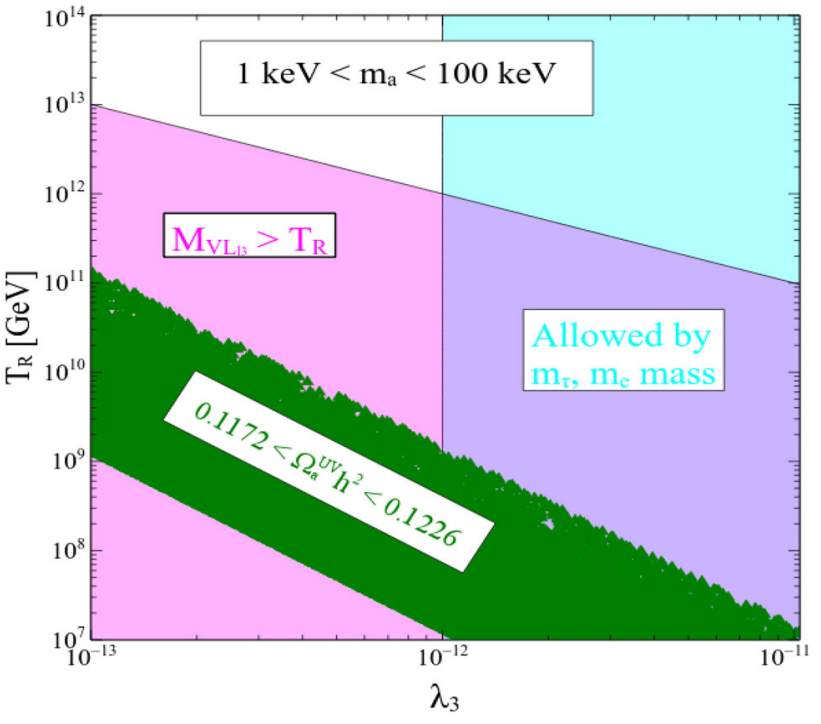

Fig. 6 Variation of reheat temperature $\left(T_{R}\right)$ with the coupling $\lambda_{3}$ for DM mass between 1 to $100 \mathrm{keV}$. Magenta region corresponds to $M_{V L_{l 3}}>T_{R}$ when $y_{3}^{V L}, y_{3}^{\phi_{1}} \sim 1$. Cyan region is allowed by the electron and tauon mass

Magenta region is coming when we impose the condition $M_{V L_{l 3}}>T_{R}$ for $y_{3}^{V L}, y_{3}^{\phi} \sim 1$, whereas the cyan region is allowed by the electron and tauon mass. All the green points satisfy the dark matter relic density put by Planck collaboration. As seen in Eq. (53), the comoving number density of the DM varies as $Y_{U V} \propto T_{R} \lambda_{3}^{2}$ (as discussed the coupling $\lambda_{1}$ is suppressed due to electron mass so we are neglecting that coupling here). Therefore, the relic density as well as the comoving number density response to $\lambda_{3}$ and $T_{R}$ parameters anti-correlated way i.e. DM relic density can be satisfied only when if $\lambda_{3}$ is increased then $T_{R}$ has to be decreased and vice versa. This type of behaviour is visible in the figure. The band of the green patch just indicates that there is also variation in the ALP mass in the range (1-100) keV. Finally, we say that the UV contribution can explain the electron mass, tauon mass, Xenon-1T from right handed signal and DM relic density altogether.

\section{Conclusion}

In this work, we have considered a $U(1)_{X}$ gauge extension of the SM gauge group, leptons and extra particles are charged and quarks are neutral under this gauge group. We have also extended the particle content by three right handed neutrinos, two vector like leptons and two singlet scalars. We have assigned the $U(1)_{X}$ as well SM gauge group charges to the particles in such a way that the model is gauge anomaly free. Due to the presence of the right handed neutrinos, we can generate the neutrino mass by the type-I seesaw mechanism. We have shown scatter plots among the neutrino mass 
parameters after satisfying the neutrino oscillation data in $3 \sigma$ range for normal hierarchy and one can extrapolate this part for the inverted hierarchy as well. The successful production of ALP dark matter from the right handed neutrino decay through freeze in mechanism has been explored. We also have two singlet scalars and among the four d.o.f, two of them act as the physical Higgses, one is absorbed by the extra gauge boson and the remaining one act like ALP. We can generate the coupling of the ALP with electron and by suitably adjusting the parameter we can explain the Xenon-1T signal as well. The global symmetry introduced is anomaly free, hence the axion coupling to photons is suppressed and can evade the CXB easily. We have considered ALP as the FIMP type DM candidate and has also been produced from the decay of the vector like leptons besides its production neutrino decay. Moreover, ALP can be produced from the higher dimensional operator as well by suitably adjusting the reheat temperature for heavy vector like lepton. We have also pointed out that if we consider decay contribution to ALP production then it is difficult to explain the lepton mass and Xenon-1T signal together. This problem can be resolved if we consider the ALP production from the higher dimen-
Data Availability Statement This manuscript has no associated data or the data will not be deposited. [Authors' comment: The datasets generated during and/or analysed during the current study are available from the corresponding author on reasonable request.]

Open Access This article is licensed under a Creative Commons Attribution 4.0 International License, which permits use, sharing, adaptation, distribution and reproduction in any medium or format, as long as you give appropriate credit to the original author(s) and the source, provide a link to the Creative Commons licence, and indicate if changes were made. The images or other third party material in this article are included in the article's Creative Commons licence, unless indicated otherwise in a credit line to the material. If material is not included in the article's Creative Commons licence and your intended use is not permitted by statutory regulation or exceeds the permitted use, you will need to obtain permission directly from the copyright holder. To view a copy of this licence, visit http://creativecomm ons.org/licenses/by/4.0/.

Funded by $\mathrm{SCOAP}^{3}$.

\section{Appendix A: Analytical expression of relevant decay} width

- $\Gamma_{N_{j} \rightarrow N_{i} a}$ :

Decay width of $N_{j}$ decaying to $N_{i} a$ is given by,

$$
\Gamma_{N_{j} \rightarrow N_{i} a}=\frac{M_{N_{j}} g_{N_{j} N_{i} a}^{2}}{16 \pi} \sqrt{\left(1-\frac{\left(M_{N_{i}}+m_{a}\right)^{2}}{M_{N_{j}}^{2}}\right)\left(1-\frac{\left(M_{N_{i}}-m_{a}\right)^{2}}{M_{N_{j}}^{2}}\right)}\left(1+\frac{M_{N_{i}}^{2}-m_{a}^{2}}{M_{N_{j}}^{2}}\right)
$$

sional operator which appear when we integrated out the vector like lepton. Since we have integrated out the vector like lepton, we have always followed that vector like lepton mass where $g_{N_{j} N_{i} a}$ is the coupling associated with $N_{j}, N_{i}$ and $a$ and is equal to $\kappa_{i j}$ as given in Eq. (21). $M_{N_{i}}$ and $m_{a}$ are the masses of the $N_{i}$ and ALP $a$.

- $\Gamma_{a \rightarrow v_{i} v_{j}}$ :

Decay width of $a$ decaying to $v_{i} v_{j}$ is given by,

$$
\Gamma_{a \rightarrow v_{i} v_{j}}=\frac{m_{a} g_{a v_{i} v_{j}}^{2}}{16 \pi} \sqrt{\left(1-\frac{\left(M_{\nu_{i}}+M_{v_{j}}\right)^{2}}{m_{a}^{2}}\right)\left(1-\frac{\left(M_{\nu_{i}}-M_{v_{j}}\right)^{2}}{m_{a}^{2}}\right)}\left(1-\frac{M_{v_{i}}^{2}+M_{v_{j}}^{2}}{m_{a}^{2}}\right)
$$

is greater than the reheat temperature of the Universe. The present model can explain the neutrino mass, Xenon-1T signal through ALP interaction with the electron. ALP can also serve as the viable DM candidate of the Universe which can be successfully produced by the freeze in mechanism either from the decay process or the annihilation process coming from higher dimensional operator.

Acknowledgements SK acknowledges the cluster computing facility at GWDG, Göttingen, Germany. SK is grateful to Laura Covi for revising the manuscript and discussion on the reheat temperature associated with the mass of vector like lepton. where $g_{a v_{i} v_{j}}^{2}$ is the coupling associated with $a, N_{i}$ and $N_{j}$. It takes the following form,

$g_{a v_{i} v_{j}}=\kappa_{m n}\left(M_{D} M_{R}^{-1}\right)_{m i}\left(M_{D} M_{R}^{-1}\right)_{n j}$,

where $\kappa_{m n}$ is given in Eq. (21). $M_{v_{i}}$ is the mass of the $v_{i}$.

\section{References}

1. Y. Sofue, V. Rubin, Rotation curves of spiral galaxies. Ann. Rev. Astron. Astrophys. 39, 137 (2001). arXiv:astro-ph/0010594 
2. D. Clowe, A. Gonzalez, M. Markevitch, Weak lensing mass reconstruction of the interacting cluster 1E0657-558: direct evidence for the existence of dark matter. Astrophys. J. 604, 596 (2004). arXiv:astro-ph/0312273

3. D. Harvey, R. Massey, T. Kitching, A. Taylor, E. Tittley, The nongravitational interactions of dark matter in colliding galaxy clusters. Science 347, 1462 (2015). arXiv:1503.07675 [astro-ph.CO]

4. M. Bartelmann, P. Schneider, Weak gravitational lensing. Phys. Rep. 340, 291 (2001). arXiv:astro-ph/9912508

5. G. Hinshaw et al. (WMAP Collaboration), Nine-year Wilkinson microwave anisotropy probe (WMAP) observations: cosmological parameter results. Astrophys. J. Suppl. 208, 19 (2013). arXiv:1212.5226 [astro-ph.CO]

6. P.A.R. Ade et al. (Planck Collaboration), Planck 2015 results. XIII. Cosmological parameters. arXiv:1502.01589 [astro-ph.CO]

7. C.L. Cowan, F. Reines, F.B. Harrison, H.W. Kruse, A.D. McGuire, Detection of the free neutrino: a confirmation. Science 124, 103 (1956)

8. Y. Fukuda et al. (Super-Kamiokande Collaboration), Evidence for oscillation of atmospheric neutrinos. Phys. Rev. Lett. 81, 1562 (1998). arXiv:hep-ex/9807003

9. Q.R. Ahmad et al. (SNO Collaboration), Direct evidence for neutrino flavor transformation from neutral current interactions in the Sudbury Neutrino Observatory. Phys. Rev. Lett. 89, 011301 (2002). arXiv:nucl-ex/0204008

10. K. Eguchi et al. (KamLAND Collaboration), First results from KamLAND: evidence for reactor anti-neutrino disappearance. Phys. Rev. Lett. 90, 021802 (2003). arXiv:hep-ex/0212021

11. F.P. An et al. (Daya Bay Collaboration), Measurement of the reactor antineutrino flux and spectrum at Daya Bay. Phys. Rev. Lett. 116(6), 061801 (2016). arXiv:1508.04233 [hep-ex]

12. J.H. Choi et al. (RENO Collaboration), Observation of energy and baseline dependent reactor antineutrino disappearance in the RENO experiment. Phys. Rev. Lett. 116(21), 211801 (2016). arXiv:1511.05849 [hep-ex]

13. Y. Abe et al. (Double Chooz Collaboration), Improved measurements of the neutrino mixing angle $\theta_{13}$ with the Double Chooz detector. JHEP 1410, 086 (2014). arXiv:1406.7763 [hepex] [Erratum: JHEP 1502, 074 (2015)]

14. K. Abe et al. (T2K Collaboration), Measurements of neutrino oscillation in appearance and disappearance channels by the T2K experiment with $6.6 \times 10^{20}$ protons on target. Phys. Rev. D 91(7), 072010 (2015). arXiv: 1502.01550 [hep-ex]

15. M. Ravonel Salzgeber (T2K Collaboration), Anti-neutrino oscillations with T2K. arXiv:1508.06153 [hep-ex]

16. P. Adamson et al. (NOvA Collaboration), First measurement of electron neutrino appearance in NOvA. Phys. Rev. Lett. 116(15), 151806 (2016). arXiv:1601.05022 [hep-ex]

17. P. Adamson et al. (NOvA Collaboration), First measurement of muon-neutrino disappearance in NOvA. Phys. Rev. D 93(5), 051104 (2016). arXiv:1601.05037 [hep-ex]

18. E. Aprile et al. (XENON), arXiv:2006.09721 [hep-ex]

19. M.M. Miller Bertolami, B.E. Melendez, L.G. Althaus, J. Isern, JCAP 10, 069 (2014). https://doi.org/10.1088/1475-7516/2014/ 10/069. arXiv:1406.7712 [hep-ph]

20. A. Ayala, I. Domínguez, M. Giannotti, A. Mirizzi, O. Straniero, Phys. Rev. Lett. 113(19), 191302 (2014). https://doi.org/10.1103/ PhysRevLett.113.191302. arXiv:1406.6053 [astro-ph.SR]

21. N. Viaux, M. Catelan, P.B. Stetson, G. Raffelt, J. Redondo, A.A.R. Valcarce, A. Weiss, Phys. Rev. Lett. 111, 231301 (2013). https:// doi.org/10.1103/PhysRevLett.111.231301. arXiv:1311.1669 [astro-ph.SR]

22. M. Giannotti, I.G. Irastorza, J. Redondo, A. Ringwald, K. Saikawa, JCAP 10, 010 (2017). https://doi.org/10.1088/ 1475-7516/2017/10/010. arXiv:1708.02111 [hep-ph]
23. L. Di Luzio, M. Giannotti, E. Nardi, L. Visinelli, arXiv:2003.01100 [hep-ph]

24. G.G. Raffelt, Phys. Lett. B 166, 402-406 (1986). https://doi.org/ 10.1016/0370-2693(86)91588-1

25. A.H. Córsico, L.G. Althaus, M.M. Miller Bertolami, S.O. Kepler, E. García-Berro, JCAP 08, 054 (2014). https://doi.org/10.1088/ 1475-7516/2014/08/054. arXiv:1406.6034 [astro-ph.SR]

26. A.H. Córsico, L.G. Althaus, M.M. Miller Bertolami, S.O. Kepler, Astron. Astrophys. Rev. 27(1), 7 (2019). https://doi.org/10.1007/ s00159-019-0118-4. arXiv:1907.00115 [astro-ph.SR]

27. S.A. Díaz, K.P. Schröder, K. Zuber, D. Jack, E.E.B. Barrios, arXiv:1910.10568 [astro-ph.SR]

28. I.M. Bloch, A. Caputo, R. Essig, D. Redigolo, M. Sholapurkar, T. Volansky, arXiv:2006.14521 [hep-ph]

29. R. Hill, K.W. Masui, D. Scott, Appl. Spectrosc. 72(5), 663-688 (2018). https://doi.org/10.1177/0003702818767133. arXiv:1802.03694 [astro-ph.CO]

30. F. Takahashi, M. Yamada, W. Yin, arXiv:2006.10035 [hep-ph]

31. K. Kannike, M. Raidal, H. Veermäe, A. Strumia, D. Teresi, arXiv:2006.10735 [hep-ph]

32. G. Alonso-Álvarez, F. Ertas, J. Jaeckel, F. Kahlhoefer, L.J. Thormaehlen, arXiv:2006.11243 [hep-ph]

33. B. Fornal, P. Sandick, J. Shu, M. Su, Y. Zhao, arXiv:2006.11264 [hep-ph]

34. C. Boehm, D.G. Cerdeno, M. Fairbairn, P.A.N. Machado, A.C. Vincent, arXiv:2006.11250 [hep-ph]

35. K. Harigaya, Y. Nakai, M. Suzuki, arXiv:2006.11938 [hep-ph]

36. L. Su, W. Wang, L. Wu, J.M. Yang, B. Zhu, arXiv:2006.11837 [hep-ph]

37. L. Di Luzio, M. Fedele, M. Giannotti, F. Mescia, E. Nardi, arXiv:2006.12487 [hep-ph]

38. Y. Chen, J. Shu, X. Xue, G. Yuan, Q. Yuan, arXiv:2006.12447 [hep-ph]

39. N.F. Bell, J.B. Dent, B. Dutta, S. Ghosh, J. Kumar, J.L. Newstead, arXiv:2006.12461 [hep-ph]

40. U.K. Dey, T.N. Maity, T.S. Ray, arXiv:2006.12529 [hep-ph]

41. J. Buch, M.A. Buen-Abad, J. Fan, J.S.C. Leung, arXiv:2006.12488 [hep-ph]

42. G. Choi, M. Suzuki, T.T. Yanagida, arXiv:2006.12348 [hep-ph]

43. D. Aristizabal Sierra, V. De Romeri, L.J. Flores, D.K. Papoulias, arXiv:2006.12457 [hep-ph]

44. G. Paz, A.A. Petrov, M. Tammaro, J. Zupan, arXiv:2006.12462 [hep-ph]

45. M. Lindner, Y. Mambrini, T.B. de Melo, F.S. Queiroz, arXiv:2006.14590 [hep-ph]

46. R. Budnik, H. Kim, O. Matsedonskyi, G. Perez, Y. Soreq, arXiv:2006.14568 [hep-ph]

47. K. Zioutas, G. Cantatore, M. Karuza, A. Kryemadhi, M. Maroudas, Y.K. Semertzidis, arXiv:2006.16907 [hep-ph]

48. L. Delle Rose, G. Hütsi, C. Marzo, L. Marzola, arXiv:2006.16078 [hep-ph]. [49]

49. C. Dessert, J.W. Foster, Y. Kahn, B.R. Safdi, arXiv:2006.16220 [hep-ph]

50. P. Coloma, P. Huber, J.M. Link, arXiv:2006.15767 [hep-ph]

51. W. Chao, Y. Gao, M. Jin, arXiv:2006.16145 [hep-ph]

52. C. Cai, H.H. Zhang, G. Cacciapaglia, M. Rosenlyst, M.T. Frandsen, arXiv:2006.16267 [hep-ph]

53. P. Ko, Y. Tang, arXiv:2006.15822 [hep-ph]

54. H. Alhazmi, D. Kim, K. Kong, G. Mohlabeng, J.C. Park, S. Shin, arXiv:2006.16252 [hep-ph]

55. S. Baek, J. Kim, P. Ko, arXiv:2006.16876 [hep-ph]

56. T. Li, arXiv:2007.00874 [hep-ph]

57. S.C. Inan, A.V. Kisselev, arXiv:2007.01693 [hep-ph]

58. K. Benakli, C. Branchina, G. Lafforgue-Marmet, arXiv:2007.02655 [hep-ph] 
59. N. Okada, S. Okada, D. Raut, Q. Shafi, arXiv:2007.02898 [hep$\mathrm{ph}]$

60. G. Choi, T.T. Yanagida, N. Yokozaki, arXiv:2007.04278 [hep-ph]

61. H. Davoudiasl, P.B. Denton, J. Gehrlein, arXiv:2007.04989 [hep$\mathrm{ph}]$

62. H.J. He, Y.C. Wang, J. Zheng, arXiv:2007.04963 [hep-ph]

63. P. Athron, C. Balázs, A. Beniwal, J.E. Camargo-Molina, A. Fowlie, T.E. Gonzalo, S. Hoof, F. Kahlhoefer, D.J.E. Marsh, M.T. Prim, P. Scott, W. Su, M. White, L. Wu, Y. Zhang, arXiv:2007.05517 [astro-ph.CO]

64. P. Anastasopoulos, K. Kaneta, Y. Mambrini, M. Pierre, arXiv:2007.06534 [hep-ph]

65. F. Arias-Aragon, F. D’Eramo, R.Z. Ferreira, L. Merlo, A. Notari, arXiv:2007.06579 [hep-ph]

66. D. Choudhury, S. Maharana, D. Sachdeva, V. Sahdev, arXiv:2007.08205 [hep-ph]

67. G. Arcadi, A. Bally, F. Goertz, K. Tame-Narvaez, V. Tenorth, S. Vogl, arXiv:2007.08500 [hep-ph]

68. F. Takahashi, M. Yamada, W. Yin, arXiv:2007.10311 [hep-ph]

69. J. Cao, X. Du, Z. Li, F. Wang, Y. Zhang, arXiv:2007.09981 [hep$\mathrm{ph}]$

70. G. Arcadi, D. Meloni, M.B. Krauss, arXiv:2007.10833 [hep-ph]

71. D. Borah, S. Mahapatra, D. Nanda, N. Sahu, arXiv:2007.10754 [hep-ph]

72. C. Han, M.L. López-Ibáãez, A. Melis, O. Vives, J.M. Yang, arXiv:2007.08834 [hep-ph]

73. S.F. Ge, P. Pasquini, J. Sheng, arXiv:2006.16069 [hep-ph]

74. B. Bhattacherjee, R. Sengupta, arXiv:2006.16172 [hep-ph]

75. K.S. Babu, S. Jana, M. Lindner, arXiv:2007.04291 [hep-ph]

76. A. Bally, S. Jana, A. Trautner, arXiv:2006.11919 [hep-ph]

77. S. Karmakar, S. Pandey, arXiv:2007.11892 [hep-ph]

78. M. Chala, A. Titov, arXiv:2006.14596 [hep-ph]

79. J. Kim, T. Nomura, H. Okada, arXiv:2007.09894 [hep-ph]

80. C.W. Chiang, B.Q. Lu, arXiv:2007.06401 [hep-ph]

81. S. Vagnozzi, L. Visinelli, P. Brax, A.C. Davis, J. Sakstein, arXiv:2103.15834 [hep-ph]

82. J. McDonald, Phys. Rev. Lett. 88, 091304 (2002). https://doi.org/ 10.1103/PhysRevLett.88.091304. arXiv:hep-ph/0106249

83. L.J. Hall, K. Jedamzik, J. March-Russell, S.M. West, JHEP 03, 080 (2010). https://doi.org/10.1007/JHEP03(2010)080. arXiv:0911.1120 [hep-ph]

84. K. Ishiwata, M.B. Wise, Phys. Rev. D 88(5), 055009 (2013). https://doi.org/10.1103/PhysRevD.88.055009. arXiv:1307.1112 [hep-ph]

85. Z. Poh, S. Raby, Phys. Rev. D 96(1), 015032 (2017). https://doi. org/10.1103/PhysRevD.96.015032. arXiv:1705.07007 [hep-ph]

86. R. Dermisek, A. Raval, Phys. Rev. D 88, 013017 (2013). https:// doi.org/10.1103/PhysRevD.88.013017. arXiv:1305.3522 [hep$\mathrm{ph}]$
87. K. Kannike, M. Raidal, D.M. Straub, A. Strumia, JHEP 02, 106 (2012). https://doi.org/10.1007/JHEP02(2012)106. arXiv:1111.2551 [hep-ph] [Erratum: JHEP 10, 136 (2012)]

88. D. Cadamuro, J. Redondo, JCAP 02, 032 (2012). https://doi.org/ 10.1088/1475-7516/2012/02/032. arXiv:1110.2895 [hep-ph]

89. R. Essig, E. Kuflik, S.D. McDermott, T. Volansky, K.M. Zurek, JHEP 11, 193 (2013). https://doi.org/10.1007/JHEP11(2013)193. arXiv:1309.4091 [hep-ph]

90. M. Ackermann et al. (Fermi-LAT), Phys. Rev. D 91(12), 122002 (2015). https://doi.org/10.1103/PhysRevD.91.122002. arXiv:1506.00013 [astro-ph.HE]

91. K. Perez, K.C.Y. Ng, J.F. Beacom, C. Hersh, S. Horiuchi, R. Krivonos, Phys. Rev. D 95(12), 123002 (2017). https://doi.org/ 10.1103/PhysRevD.95.123002. arXiv:1609.00667 [astro-ph.HE]

92. C. Blanco, D. Hooper, JCAP 03, 019 (2019). https://doi.org/10. 1088/1475-7516/2019/03/019. arXiv:1811.05988 [astro-ph.HE]

93. F. Capozzi, E. Lisi, A. Marrone, D. Montanino, A. Palazzo, Nucl. Phys. B 908, 218-234 (2016). https://doi.org/10.1016/j. nuclphysb.2016.02.016. arXiv:1601.07777 [hep-ph]

94. E. Giusarma, M. Gerbino, O. Mena, S. Vagnozzi, S. Ho, K. Freese, Phys. Rev. D 94(8), 083522 (2016). https://doi.org/10. 1103/PhysRevD.94.083522. arXiv:1605.04320 [astro-ph.CO]

95. S. Vagnozzi, E. Giusarma, O. Mena, K. Freese, M. Gerbino, S. Ho, M. Lattanzi, Phys. Rev. D 96(12), 123503 (2017). https://doi.org/ 10.1103/PhysRevD.96.123503. arXiv:1701.08172 [astro-ph.CO]

96. K. Nakayama, F. Takahashi, T.T. Yanagida, Phys. Lett. B 734, 178-182 (2014). https://doi.org/10.1016/j.physletb.2014.05.035. arXiv: 1403.7390 [hep-ph]

97. E. Masso, J. Redondo, JCAP 09, 015 (2005). https://doi.org/10. 1088/1475-7516/2005/09/015. arXiv:hep-ph/0504202

98. E. Masso, J. Redondo, Phys. Rev. Lett. 97, 151802 (2006). https://doi.org/10.1103/PhysRevLett.97.151802. arXiv:hep-ph/0606163

99. J. Jaeckel, E. Masso, J. Redondo, A. Ringwald, F. Takahashi, Phys. Rev. D 75, 013004 (2007). https://doi.org/10.1103/PhysRevD.75. 013004. arXiv:hep-ph/0610203

100. A.K. Ganguly, P. Jain, S. Mandal, S. Stokes, Phys. Rev. D 76, 025026 (2007). https://doi.org/10.1103/PhysRevD.76.025026. arXiv:hep-ph/0611006

101. J.E. Kim, Phys. Rev. D 76, 051701 (2007). https://doi.org/10. 1103/PhysRevD.76.051701. arXiv:0704.3310 [hep-ph]

102. P. Brax, C. van de Bruck, A.C. Davis, Phys. Rev. Lett. 99, 121103 (2007). https://doi.org/10.1103/PhysRevLett.99.121103. arXiv:hep-ph/0703243

103. J. Redondo, arXiv:0807.4329 [hep-ph] 